\title{
Supersymmetric and non-supersymmetric models without catastrophic Goldstone bosons
}

\author{
Johannes Braathen ${ }^{1,2, a}$, Mark D. Goodsell ${ }^{1,2}$, Florian Staub ${ }^{3,4}$ \\ ${ }^{1}$ LPTHE, UPMC Univ. Paris 6, Sorbonne Universités, 4 Place Jussieu, 75252 Paris, France \\ ${ }^{2}$ LPTHE, CNRS, 4 Place Jussieu, 75252 Paris, France \\ ${ }^{3}$ Institute for Theoretical Physics (ITP), Karlsruhe Institute of Technology, Engesserstraße 7, 76128 Karlsruhe, Germany \\ ${ }^{4}$ Institute for Nuclear Physics (IKP), Karlsruhe Institute of Technology, Hermann-von-Helmholtz-Platz 1, 76344 Eggenstein-Leopoldshafen, \\ Germany
}

Received: 11 July 2017 / Accepted: 14 October 2017 / Published online: 10 November 2017

(C) The Author(s) 2017. This article is an open access publication

\begin{abstract}
The calculation of the Higgs mass in general renormalisable field theories has been plagued by the socalled "Goldstone Boson Catastrophe," where light (wouldbe) Goldstone bosons give infra-red divergent loop integrals. In supersymmetric models, previous approaches included a workaround that ameliorated the problem for most, but not all, parameter space regions; while giving divergent results everywhere for non-supersymmetric models! We present an implementation of a general solution to the problem in the public code SARAH, along with new calculations of some necessary loop integrals and generic expressions. We discuss the validation of our code in the Standard Model, where we find remarkable agreement with the known results. We then show new applications in Split SUSY, the NMSSM, the Two-Higgs-Doublet Model, and the Georgi-Machacek model. In particular, we take some first steps to exploring where the habit of using tree-level mass relations in nonsupersymmetric models breaks down, and show that the loop corrections usually become very large well before naive perturbativity bounds are reached.
\end{abstract}

\section{Introduction}

The Large Hadron Collider has opened a new era of precision physics. Following the discovery of the Higgs, the measurement of its properties - in particular its mass - have now been performed with an astonishing precision. This is interesting because a precise determination of the Higgs mass is of crucial importance in understanding the fate of the Standard Model (it is used to calculate the Higgs quartic coupling, required to determine whether the electroweak vacuum is metastable) and is especially sensitive to new physics beyond

a e-mail: braathen@1pthe.jussieu.fr the SM (BSM). This is particularly important in supersymmetric models, where there is a prediction for the Higgs quartic coupling at tree level in terms of other fundamental parameters of the theory (notably the gauge couplings). There is therefore a long tradition of calculating higher-order corrections to the Higgs mass which was founded at the beginning of the 1990s when the dominant one-loop corrections in the minimal supersymmetric standard model (MSSM) were calculated [1-3]. Nowadays, the dominant two- and even threeloop corrections are available for the MSSM in the gaugeless limit, with vanishing external momenta [4-24] and the dominant momentum-dependent two-loop corrections were given in [25-27].

However, all of these higher-order corrections bypass an intrinsic technical problem of divergences associated with would-be Goldstone bosons of the broken electroweak symmetry. Calculations beyond the Standard Model at two loops and higher have only been performed in Landau gauge in order to decouple ghosts and thus simplify the calculations; however, in this gauge the would-be Goldstone bosons are massless and lead to infra-red divergences. In the MSSM, the gaugeless limit avoids this by turning off the Goldstone boson ${ }^{1}$ couplings to the Higgs; and the other (momentumdependent) calculations that have been performed beyond this limit only consider the sector of the theory without the Goldstones.

However, as soon as one considers non-minimal supersymmetric models in which trilinear interactions of the Higgs superfields occur in the superpotential, the gaugeless limit no longer offers much protection against the problem, since the quartic coupling is not determined by the gauge couplings;

\footnotetext{
${ }^{1}$ We shall drop the prefix "would-be" from now on; although in the gaugeless limit this distinction is irrelevant because they become physical Goldstone bosons.
} 
and this is a generic feature of non-supersymmetric models (such as the Standard Model!). The so-called "Goldstone Boson Catastrophe" was noticed in the first attempt to go beyond the gaugeless limit in the MSSM at more than one loop [15], and leads to divergent values for the Higgs mass at two loops and beyond - it can in fact be a complete obstacle to a precise calculation.

Recently a solution was proposed in the context of the Standard Model [28,29] (see also [30-32] for recent related work) and then extended to the MSSM [33] which involved resumming (a subset of) the Goldstone boson propagators. An alternative for the Standard Model based on the 2PI effective action was proposed in [34-36], where essentially all particle propagators are resummed. However, both of these approaches are difficult to generalise. Instead, in [37] a general procedure was developed to cure this problem in twoloop Higgs mass calculations, based on setting the Goldstone boson propagators on-shell, which provided a complete set of modified loop functions for the tadpoles and self-energies that were finite. Thus, combining the results of [37] with those of [38-41] which provide fully generic expressions for the two-loop corrections to real scalar masses in supersymmetric and non-supersymmetric models, all ingredients are present to calculate Higgs masses in any renormalisable model.

The generic expressions of [38-41] are already used by the Mathematica package SARAH [42-47] to calculate in combination with SPheno $[48,49]$ the Higgs masses in supersymmetric models at the two-loop level [41,50,51]. Up to now, the workaround for the Goldstone boson catastrophe in this setup was to introduce finite masses for the electroweak Goldstones by dropping the D-terms in the mass matrices. However, there were many regions of parameter space where the divergences reappeared (see e.g. [52-54]) and this does not work at all for non-supersymmetric models, which have no D-term potential! Therefore, to perform this work we have implemented the results of [37], in addition to filling some additional technical gaps which we describe here in Sect. 2 and the appendices; in particular, we complete the basis of required loop functions. The new version of SARAH 4.12 .0 therefore now offers the possibility to calculate two-loop masses for neutral scalars in non-supersymmetric models, as well as substantially improving the calculation in supersymmetric ones. As the only non-supersymmetric model for which comparable results exist is the Standard Model, in Sect. 3 we compare our new calculation against the public code SMH [55] and the results of [56], finding excellent agreement (even if our results do not include all of the contributions included in those references). We then illustrate our new routines by computing some new results in Split SUSY in Sect. 5. On the other hand, in Sect. 4 we show how our new approach improves our previous calculation for supersymmetric models through the example of the
NMSSM, for which our results should now be considered state of the art.

Momentum-independent renormalisation schemes are the most convenient choices for applying to a large variety of models, and so all mass calculations in SARAH are performed in the $\overline{\mathrm{MS}}$ or $\overline{\mathrm{DR}}^{\prime}$ scheme. In contrast, on-shell schemes might offer some model dependent advantages. This is for instance the case in supersymmetric models with Dirac gauginos and a large mass splitting between the stops and the gluino. It has been shown that in this case an on-shell scheme leads to an improved convergence of the perturbative series [57]. It is also very useful often if a $\overline{\mathrm{DR}}^{\prime}$ and on-shell calculation exists for the same supersymmetric model: the difference between the results can be used as estimate of the missing higher-order corrections; this can now be done for the MSSM and certain classes of NMSSM and Dirac gaugino contributions. On the other hand, there has been hardly any discussion in the literature about radiative corrections to Higgs masses in non-supersymmetric BSM models. One reason for this, besides the technical hurdles, is that the additional freedom in non-supersymmetric models introduces a large number of free parameters, i.e. in some cases it might be possible to absorb any finite correction in the scalar sector into the counter-terms of these parameters. Thus, it is often implicitly assumed that the masses, but also the mixing angles, in the extended Higgs sector in BSM could be kept at their tree-level values. However, this is fraught with danger: (1) not all non-supersymmetric models really have a sufficiently large number of free parameters to absorb all radiative corrections. This is for instance the case in the GeorgiMachacek model. (2) If a low-energy model is combined with an explicit UV completion (such as a GUT theory), the freedom to adjust the couplings is usually lost. (3) Using masses instead of couplings as input hides the presence of huge or even non-perturbative quartic couplings. (4) Even if parameters are checked with respect to simple limits such as $\lambda<4 \pi$ or tree-level unitarity bounds, this does not guarantee that the considered parameter point is perturbative or that strongly coupled effects do not appear at lower energies than can be explored at the LHC. Partly motivated by the growing interest in exploring quantum corrections to nonsupersymmetric models, here in Sects. 6 and 7 we explore the corrections to the Two-Higgs-Doublet Model (2HDM) and Georgi-Machacek model (GM), drawing attention to the fact that the corrections pass out of control well before the naive perturbativity or unitarity bounds.

Finally, an $\overline{\mathrm{MS}}$ calculation has the advantage that it can give an impression of the size of the theoretical uncertainty by varying the renormalisation scale. Moreover, to obtain more reliable results for the vacuum stability by considering the renormalisation group equation (RGE) improved effective potential, a translation of masses into $\overline{\mathrm{MS}}$ parameters is necessary. We show in this work how these aspects can be 
analysed in non-supersymmetric models with the new calculation available now in SARAH.

\section{The Goldstone Boson Catastrophe and its solutions}

To calculate the Higgs boson masses in general field theories we require the tadpole diagrams and self-energies. Expressions for the former were given in [41], which were derived from the general expression for the effective potential in the Landau gauge, given in [58]. Hence we must also use the selfenergies in the Landau gauge; these were given in [38] up to order $g^{2}$ in the gauge couplings, and so we restrict ourselves to the "gaugeless limit" where we ignore the contributions of broken gauge groups. This has a number of advantages, chiefly simplicity and speed of the calculation; but also the fact that we can compute the one-loop corrections in any gauge desired. Once we have dropped the electroweak contributions, it is also tempting to disregard the momentum dependence of the loop functions, which is typically estimated to contribute at the same order (and indeed is so for the MSSM [26,27]) - hence the popularity of calculations in the effective potential approach.

However, calculations in the Landau gauge/gaugeless limit suffer from the "Goldstone Boson Catastrophe", where the Goldstone bosons lead to ill-defined or divergent loop functions. Let us define the scalar potential in terms of real scalar fields $\varphi_{i}^{0}$ and their fluctuations around expectation values $v_{i}$ such that $\varphi_{i}^{0} \equiv v_{i}+\phi_{i}^{0}$ (not necessarily mass diagonal):

$$
\begin{aligned}
V^{(0)}\left(\left\{\varphi_{i}^{0}\right\}\right)= & \frac{1}{2} m_{0, i j}^{2} \varphi_{i}^{0} \varphi_{j}^{0}+\frac{1}{6} \lambda_{0}^{i j k} \varphi_{i}^{0} \varphi_{j}^{0} \varphi_{k}^{0} \\
& +\frac{1}{24} \lambda_{0}^{i j k l} \varphi_{i}^{0} \varphi_{j}^{0} \varphi_{k}^{0} \varphi_{l}^{0} \\
= & V^{(0)}\left(v_{i}\right)+t^{i} \phi_{i}^{0}+\frac{1}{2} m_{i j}^{2} \phi_{i}^{0} \phi_{j}^{0} \\
& +\frac{1}{6} \hat{\lambda}_{0}^{i j k} \phi_{i}^{0} \phi_{j}^{0} \phi_{k}^{0}+\frac{1}{24} \hat{\lambda}_{0}^{i j k l} \phi_{i}^{0} \phi_{j}^{0} \phi_{k}^{0} \phi_{l}^{0},
\end{aligned}
$$

where $t^{i}$ are tadpoles. Since we define the VEVs to be exact, we must have

$t^{i}+\left.\frac{\partial \Delta V\left(\left\{m_{i j}^{2}\right\}\right)}{\partial \phi_{i}^{0}}\right|_{\phi_{i}^{0}=0}=0$.

By defining the potential in terms of fluctuations, we have the $\overline{\mathrm{MS}} / \overline{\mathrm{DR}}$ masses squared $m_{i j}^{2}$ for all the scalars in the theory, and these are the values that enter the loop functions. However, the tadpoles are functions of the masses:

$t^{i}=m_{0, i j}^{2} v_{j}+\frac{1}{2} \lambda_{0}^{i j k} v_{j} v_{k}+\frac{1}{6} \lambda_{0}^{i j k l} v_{j} v_{k} v_{l}$ and so, since these need to be adjusted loop order by order, we must choose some parameters to vary - and the standard choice is the mass-squared parameters, because in this way the couplings are unaffected. So then we define

$m_{i j}^{2} \equiv \hat{m}_{0, i j}^{2}+\Delta_{i j}$

where $\hat{m}_{0, i j}^{2}$ is the value without loop corrections (so $t^{i}=0$ ) and $m_{i j}^{2}$ satisfies the full tadpole equations:

$m_{i j}^{2} v_{j}=\hat{m}_{0, i j}^{2} v_{j}-\left.\frac{\partial \Delta V\left(\left\{m_{i j}^{2}\right\}\right)}{\partial \phi_{i}^{0}}\right|_{\phi_{i}^{0}=0}$,

where $\Delta V$ consists of the loop corrections to the effective potential, and we have written explicitly its dependence on the parameters $\left\{m_{i j}^{2}\right\}$. The Goldstone Boson Catastrophe appears because the mass-squared parameter(s) of the Goldstone boson(s) in the Lagrangian is (are) zero at tree level, but non-zero once we take into account the loop corrections to the potential. Then at two loops and higher we must calculate loop corrections with a small and/or negative mass-squared parameter, which leads to large logarithms and/or phases.

In the context of the Standard Model [28,29] (see also [30-32]) and MSSM [33] it was suggested that resumming (a subset of) the Goldstone boson self-energies would cure divergences in the tadpole diagrams; and including external momenta in the self-energies would also be required to cure divergences there. Alternatively, Refs. [3436] proposed using the (symmetry-improved) two-particleirreducible potential to cure the problem in the Standard Model, which provides a consistent theoretical underpinning but unfortunately is particularly difficult to generalise. In the following we shall describe our previous approaches to the problem and the new results and implementation in SARAH.

\subsection{Previous approaches in SARAH}

Up until now, in SARAH the catastrophe appeared in an even more acute form because all of the one- and two-loop tadpoles and self-energies are computed using the tree-level masses in the loops, so without a solution to the problem, the Goldstone bosons are massless and cause several loop functions to diverge. However, for supersymmetric models the original workaround implemented in $[41,50]$ and explored in more detail in [51] relies on the fact that the electroweak gauge couplings appear in the D-term potential. ${ }^{2}$ We therefore used the tree-level parameters that are solutions of the full tree-level tadpole equations including the electroweak

\footnotetext{
2 Indeed, the gaugeless limit (turning off the electroweak gauge couplings) completely cures the problem in the MSSM by eliminating all of the Goldstone boson couplings to the Higgs.
} 
couplings to calculate the tree-level masses (but set the electroweak gauge couplings to zero in the mass matrices) used in the two-loop routines' loop functions. In other words, the masses in the loop functions are not at the minimum of the potential, and are typically tachyonic, ${ }^{3}$ with a size of order the electroweak scale. Since we are neglecting two-loop corrections proportional to these couplings, this error is acceptable. On the other hand, for models beyond the MSSM (in particular, the NMSSM) there are typically regions of the parameter space where the Higgs sector masses still pass near to zero and cause the loop functions to diverge; for example such problems were observed in [52-54].

A more recent approach was to introduce regulator masses. All scalar masses in the two-loop routines which are below a certain threshold are set in terms of the renormalisation scale $Q$ and a constant $R$ :

$m_{S, \min }^{2}=R Q^{2}$

This approach was introduced in SARAH to stabilise cases in which the D-term approach fails. This could either be, as demonstrated in an example in Sect. 4, if other scalars artificially become very light, or if the supersymmetric scale is much higher than the electroweak scale. However, in contrast to the D-term solution, this approach violates the symmetries of the theory and can lead to non-zero masses for Goldstone bosons. Furthermore, there is no a priori indication for the optimal size of $R$; too large and the Goldstone/Higgs contributions are suppressed (because logarithmic contributions including them are artificially reduced), too small and the results become numerically unstable, and the user must use trial and error. Finally, it implicitly assumes that the corrections coming from the Higgs/Goldstone bosons to the Higgs mass are small (so that modifying them is benign). This is not a good approximation in many non-SUSY models, and for this reason the newly implemented solution described in the next section allows non-SUSY models to be studied accurately for the first time.

\subsection{On-shell Goldstone bosons and consistent tadpole solutions}

In [37] a genuine solution was presented for generic field theories: we should treat the Goldstone boson mass as an onshell parameter. A set of modified expressions for tadpoles and self-energies were given -indeed, it was shown that there were a class of loop diagrams that were not made finite purely by including external momenta. In addition, expressions for the "consistent solution" of the tadpole equations were given.

\footnotetext{
3 Since the mass was tachyonic and generally not small, we then neglected the imaginary part of the self-energies/tadpoles.
}

These two results are closely related, as we shall elaborate a little here.

If we take the Goldstone boson mass on-shell, as proposed in [37], then we have two possible ways of calculating the resulting tadpoles and self-energies, which differ in terms of how we solve the tadpole equations. The choice arises because the mass parameters $m_{i j}^{2}$ appear on both the leftand the right-hand sides of Eq. (2.5), so we can:

1. Numerically solve Eq. (2.5) to find the $m_{i j}^{2}$ exactly.

2. Perturbatively expand the $m_{i j}^{2}$ so that

$$
m_{i j}^{2}=\hat{m}_{0, i j}^{2}+\delta^{(1)} m_{i j}^{2}+\delta^{(2)} m_{i j}^{2}+\cdots
$$

and solve for a given loop order.

Since the effective potential $\Delta V$ will only be computed to a given loop order, the two approaches are formally equivalent. For the first approach, in practice, this means that we must iteratively solve the tadpole equations; at each iteration we put $m_{i}^{2}=R_{k i} R_{l i} m_{k l}^{2}$ for the tree-level mass parameters, computing a new $R$ each time and therefore modifying the couplings, and then set the Goldstone boson mass to zero in the loop functions and compute the tadpole equations from the expressions in [37]. We find in this case that the couplings are no longer guaranteed to satisfy certain relationships imposed by the broken symmetries; only the full onshell amplitudes will satisfy the appropriate Slavnov-Taylor identities. This is only a problem for the coupling $\lambda^{G G^{\prime} G^{\prime \prime}}$ between three Goldstone bosons, which is zero at tree level and on-shell; because the parameter in the Lagrangian will in general obtain a small non-zero value (in theories with CP-violation) and yet leads to divergent Goldstone boson self-energies we must impose that this is also on-shell (i.e. zero). Since this coupling does not appear at one loop in the calculation of the Higgs boson mass, taking this coupling to vanish causes no shift at two loops.

On the other hand, if we want to calculate the Goldstone boson self-energy at two loops then we $d o$ find a set of shifts when we take this coupling "on-shell": we would need to include the vertex corrections and define a set of shifted loop functions for those contributions (which, of course, only affect the self-energies). We shall return to this in future work.

Instead, in our implementation of the results of [37] in SARAH we take the second approach in the list above: we expand $m_{i j}^{2}$ as a series in the couplings, and solve explicitly up to two-loop order in one step without recursion. This was already proposed in [33] for the MSSM, and in [37] explicit formulae for the corrections to the tadpoles and masses with a so-called consistent tadpole solution were given for the general case. Then we can calculate all of our loop functions using the masses $\left(\hat{m}_{0, i j}^{2}\right)$ and couplings in the tree-level Lagrangian, and shifts $\Delta_{i j}$. 
However, here we shall also generalise a little the expressions given in [37]: we shall allow $\Delta_{i j}$ to be an implicit function of the tadpole shifts, rather than explicitly assuming $\Delta_{i j}=-\left.\delta_{i j} \frac{1}{v_{i}} \frac{\partial \Delta V}{\partial \phi_{i}^{0}}\right|_{\phi_{i}^{0}=0}$; indeed, this equation fails for pseudoscalars, for example. Instead we solve the tadpole equations for some variables $\left\{x_{i}\right\}$ with

$x_{i}=c_{0, i}+c_{i j} \times \frac{\partial \Delta V}{\partial \phi_{j}^{0}}$

then

$\Delta_{i j}=\sum_{k, l} \frac{\partial m_{i j}^{2}}{\partial x_{k}} c_{k l} \frac{\partial \Delta V}{\partial \phi_{l}^{0}}$

For example, in the Goldstone model of a single complex scalar $\Phi$ having potential

$V=\mu^{2}|\Phi|^{2}+\lambda|\Phi|^{4}$

when $\Phi$ obtains a VEV it decomposes into a real scalar $h$ and a Goldstone boson $G$ as $\Phi=\frac{1}{\sqrt{2}}(v+h+i G)$. We solve the tadpole equations for the parameter $\mu^{2}$ so that

$\mu^{2}+\lambda v^{2}+\frac{1}{v} \frac{\Delta V}{\partial h}=0$

However, both the mass of the Goldstone boson and the Higgs are controlled by the $\mu^{2}$ parameter;

$m_{h h}^{2}=\mu^{2}+3 \lambda v^{2}, \quad m_{G G}^{2}=\mu^{2}+\lambda v^{2}$.

So in our notation, $x_{h} \rightarrow \mu^{2}, c_{0, h} \rightarrow-\lambda v^{2}, c_{h h} \rightarrow-\frac{1}{v}$ and so

$\Delta_{h h}=-\frac{1}{v} \frac{\Delta V}{\partial h}=\Delta_{G G}$.

Substituting the $\Delta_{i j}$ into the one-loop tadpole and self-energy expressions then gives a set of two-loop shifts; for scalars these were given in Eqs. (5.2) and (5.3) of [37].

We have implemented this under the assumption that the variables $\left\{x_{i}\right\}$ are dimensionful and there is no explicit dependence of the trilinear/quartic couplings on them (only implicitly through the mixing matrices $R$ ); and also we assume that the fermion mass matrices do not depend on these parameters. These assumptions are fulfilled e.g. for $\left\{m_{H_{u}}^{2}, m_{H_{d}}^{2}\right\}$ in the MSSM, but not $\left\{\mu, B_{\mu}\right\}$ chosen as parameters to solve the tadpole equations. On the other hand, we give expressions for the shifts to the tadpoles and self-energies when fermion masses depend on the $\left\{x_{i}\right\}$ in Appendix B, and we plan to implement these in the future.
Now, since the Goldstone boson is massless at tree level, this then means that we automatically have the Goldstone boson "on-shell." This means that the "on-shell" and "consistent solution" approaches are more closely related than first appears: since the Goldstone boson mass must be zero on-shell and we can identify the Goldstone boson eigenstates using a matrix $R_{k G}$ derived just from the broken symmetries (see e.g. [37]) then the on-shell condition becomes

$$
\begin{aligned}
& \operatorname{det}\left(p^{2}-m_{i j}^{2}-\Pi_{i j}\left(p^{2}\right)\right) \\
& \quad=0 \rightarrow R_{k G} R_{l G} m_{k l}^{2}+\Pi_{G G}(0)=0 ;
\end{aligned}
$$

and since $\hat{m}_{0, G G}^{2}=0$ we have

$$
\begin{aligned}
\delta m_{G}^{2} & =R_{k G} R_{l G} m_{k l}^{2}=-\Pi_{G G}(0) \\
\Delta_{G G} & =\delta m_{G}^{2}+\mathcal{O}(2-\text { loop }) \rightarrow \Delta_{G G} \\
& =-\Pi_{G G}(0)+\mathcal{O}(2-\text { loop }),
\end{aligned}
$$

i.e. the approach of adjusting the loop functions (as we do when setting the Goldstone boson on-shell) or defining a set of shifts to the tadpoles and self-energies involving $\Delta_{i j}$ should give the same result when we just consider the shifts to the Goldstone boson masses, even though the expressions look very different.

2.3 A complete basis of loop functions and the implementation in SARAH

For the evaluation of tadpoles and self-energies [37] proposed a "generalised effective potential limit," where the selfenergies are expanded in $s=-p^{2}\left(=m^{2}\right.$ on-shell) and all terms of order $\mathcal{O}(s)$ are neglected (but crucially retaining terms that diverge at $s=0)$. We therefore require the following basis of loop functions, where $\{x, y, z, u, v\} \neq 0$ are masses squared:

Momentum independent:

$$
\begin{aligned}
& J(x), P_{S S}(x, y), P_{S S}(0, y), I(x, y, z), I(0, y, z), I(0,0, z), \\
& U_{0}(x, y, z, u), U_{0}(0, y, z, u), U_{0}(x, y, 0, u), U_{0}(0, y, 0, u), \\
& \quad U_{0}(x, y, 0,0), U_{0}(0, y, 0,0), \\
& M_{0}(x, y, z, u, v), M_{0}(0, y, z, u, v), M_{0}(0,0, z, u, v), \\
& \quad M_{0}(0,0,0, u, v), \\
& \tilde{V}(x, y, z) .
\end{aligned}
$$

Momentum dependent:

$B(0,0)$,

$M(x, 0,0,0,0), M(0, y, 0, u, v), M(0,0,0, u, v)$, $M(0,0,0,0, v)$

$U(0,0, x, y), U(0,0,0, y)$

$\tilde{V}(0, y, z), \tilde{V}(0,0, z)$. 
All of these functions are implicitly dependent on the renormalisation scale $Q$, typically containing factors of $\overline{\log } x \equiv$ $\log \left(x / Q^{2}\right)$. Expressions for all of these functions expanded up to $\mathcal{O}(1)$ in the external momenta (or the reference for them) were given in [37]. However, the functions $\tilde{V}(x, y, z)$ were given in terms of the regularised function $\bar{V}(u, 0, y, z)$ defined in [38]; ${ }^{4}$ in Appendix A we derive explicit compact expressions for this function - first with full momentum dependence, and then expanded up to $\mathcal{O}(1)$ in the external momenta.

In our practical implementation in SARAH we have extended the available routines for calculating two-loop integrals with the missing ingredients to address the Goldstone boson catastrophe. Moreover, there are three loop functions involving fermions and gauge bosons which needed modification for the $\overline{\mathrm{MS}}$ scheme as used for non-supersymmetric models, as compared to the $\overline{\mathrm{DR}}^{\prime}$ for supersymmetric models; the tadpole and self-energies contain

$$
\begin{aligned}
\frac{\partial \hat{V}^{(2)}}{\partial \phi_{r}^{0}} \supset R_{r p} & T_{F V}^{p}, \\
T_{F V}^{p}= & g^{2} d(I) C(I) \operatorname{Re}\left(M_{I I^{\prime}} y^{I I^{\prime} r}\right) \\
& \times\left(\frac{1}{2} F_{F V}^{\prime}(x)\right), \\
\Pi_{i j}^{(2)} \supset \Pi_{i j}^{F V}= & g^{2} d(K) C(K) \\
\times & {\left[\operatorname{Re}\left(y^{i K L} y_{j K L}\right) G_{F F}\left(m_{K}^{2}, m_{L}^{2}\right)\right.} \\
& +\operatorname{Re}\left(y^{i K L} y^{j K^{\prime} L^{\prime}} M_{K K^{\prime}} M_{L L^{\prime}}\right) \\
& \left.\times G_{\overline{F F}}\left(m_{K}^{2}, m_{L}^{2}\right)\right],
\end{aligned}
$$

$$
\begin{aligned}
& \left.-(x+y)\left(2 B(x, y)+x B\left(y, x^{\prime}\right)+y B\left(x, y^{\prime}\right)\right)\right] \\
\underset{s \rightarrow 0}{\rightarrow} & {\left[2 ( x + y ) \left[3 U_{0}(x, y, x, 0)+3 U_{0}(x, y, y, 0)\right.\right.} \\
& \left.+5 P_{S S}(x, y)\right]-6 I(x, x, 0)-6 I(y, y, 0) \\
& +10 J(x)+10 J(y)-16(x+y)] \\
& +4 \delta_{\overline{M S}}\left[x+y+J(x)+J(y)+(x+y) P_{S S}(x, y)\right], \\
G_{\overline{F F}}(x, y)= & G \overline{\overline{D R}}^{\prime}(x, y) \\
& -4 \delta_{\overline{M S}}\left[2 B(x, y)+y B\left(x, y^{\prime}\right)+x B\left(y, x^{\prime}\right)\right] \\
\rightarrow & 4\left(3 U_{0}(x, y, x, 0)+3 U_{0}(x, y, y, 0)+5 P_{S S}(x, y)-4\right) \\
& +4 \delta_{\overline{M S}}\left[2 P_{S S}(x, y)+1\right] .
\end{aligned}
$$

Here $\delta_{\overline{\mathrm{MS}}}$ is one for $\overline{\mathrm{MS}}$ masses and zero for $\overline{\mathrm{DR}}^{\prime}$.

In addition, routines to calculate the consistent tadpole solution are generated during the output of SPheno code. This is fully automatised beginning with SARAH version 4.12.0 and the user can obtain a SPheno version for nonsupersymmetric models as before - with the difference that two-loop mass corrections are now included. We refer for more detailed explanations of how to use the code to the standard references such as [47]. The only requirements are recent versions of SARAH and SPheno which are available at http: / / www . hepforge.org.

The new features can now be adjusted in the Block SPHENOINPUT in the Les Houches input file:

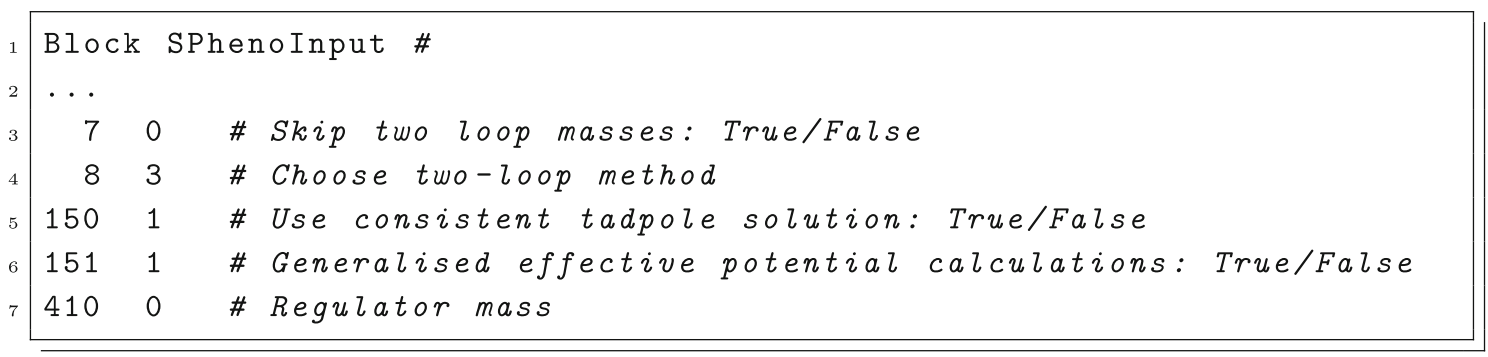

where $\hat{V}^{(2)}$ is the two-loop contribution to the effective potential, $d(I), C(I)$ are the dimension and quadratic Casimirs of representation $I$ of the gauge group having coupling $g$, and the loop functions are

$$
\begin{aligned}
\left(\frac{1}{2} F_{F V}^{\prime}(x)\right) & =4 x\left[6-7 \overline{\log } x+3 \overline{\log }^{2} x+\delta_{\overline{\mathrm{MS}}}[2 \overline{\log } x-1]\right], \\
G_{F F}(x, y) & =G_{F F}^{\overline{\mathrm{DR}}^{\prime}}(x, y)+2 \delta_{\overline{M S}}[x+y+2 J(x)+2 J(y)
\end{aligned}
$$

\footnotetext{
$\overline{{ }^{4} \text { Note that our }} \tilde{V}$ uses one fewer variable than the definition of $V$ in [38] because in our case it always appears in the form $\bar{V}(u, 0, y, z)$.
}

Note that the solution to the Goldstone boson catastrophe exists only for the diagrammatic calculation (flag $8 \rightarrow 3$ ), but not for the effective potential calculations using numerical derivatives to obtain the tadpoles and self-energies (flag $8 \rightarrow$ $1,2)$. By default, the new calculation is used now, but could be turned off if demanded (flag $151 \rightarrow 0$ ). In this case, it is usually necessary to include a non-zero regulator mass via flag 410 for non-supersymmetric models. In principle, there should not be any reason to revert to the old calculation with regulator masses except for double-checking the result.

The consistent tadpole solution (described in the previous section) is turned off by default but can be turned on 
by setting flag $150 \rightarrow 1$. This is because, while strictly it is more accurate to include it, there is also the possibility of numerical instability if the shift in the tree-level mass parameters is large; for example, if the expectation values of some scalars are small (such as e.g. the neutral scalar of an electroweak triplet which must have a small expectation value from electroweak precision constraints) then the shift in the mass parameter can be much larger than the tree-level value and the perturbative solution fails. In such cases, it would be better to use a recursive approach which is currently not possible for the reasons given in Sect. 2.2.

\section{Standard Model}

\subsection{A first comparison of our results with existing calculations}

Now that two-loop corrections to scalar masses are available in SARAH, free of the Goldstone boson catastrophe, it is important to compare the results we obtain with other computations available in the literature, as a verification of our results and as a way to estimate the impact of missing corrections. We consider in this section the Higgs mass calculations in the Standard Model, and we will compare the results obtained with SPheno with the computations performed at complete two-loop calculation in [56], and the full two-loop (plus leading three-loop) Higgs mass calculation implemented in the public code SMH [55]. These works take into account two-loop electroweak corrections, which are not available for generic theories and are not included in our code, hence we will quantify the size of these effects, together with effects from momentum, and investigate the discrepancy in masses coming from the different determination of the top Yukawa coupling.

It is interesting to examine the way that the two calculations avoid the Goldstone Boson Catastrophe. The calculation of Buttazzo et al. [56] was performed in Feynman gauge and using certain parameters on-shell, whereas the results implemented in SMH are in a pure $\overline{\mathrm{MS}}$ scheme and Landau gauge, which is closer to our approach. In the latter paper, some resummation is performed by hand to eliminate the divergence in the mass calculation; it is perhaps surprising that the absence of the function $\bar{V}(0,0, y, z)$ from the basis in TSIL was not problematic, but there the calculation was performed by computing the set of integrals explicitly using TARCER [60] rather than starting from a set of generic expressions, so the result was found directly in terms of the other basis functions. In principle this should agree with Eq. (A.10).

For clarity, we recall that we define the (tree-level) Higgs potential as

$$
V^{(0)}=-\mu^{2}|H|^{2}+\lambda|H|^{4}, \quad H=\left(\begin{array}{c}
G^{+} \\
\frac{1}{\sqrt{2}}(v+h+i G)
\end{array}\right) .
$$

A first approach for the comparison between SPheno and [56] is to compute the Higgs mass with the quartic coupling $\lambda$ ranging in the interval $[0.125,0.130]$, and only setting the SM inputs to the same values as in [56], which we recall here

$$
\begin{aligned}
G_{F} & =1.16638 \times 10^{-5} \mathrm{GeV}^{-2}, \\
\alpha_{s}\left(M_{Z}\right) & =0.1184, \\
M_{Z} & =91.1876 \mathrm{GeV}, \\
m_{t} & =173.34 \mathrm{GeV} .
\end{aligned}
$$

They furthermore took the experimentally determined central value of the Higgs mass to be $125.15 \mathrm{GeV}$, which we shall take as a reference value rather than an input. The use of consistent solutions to the tadpole equations - as derived in [37] - has also been implemented in the SPheno code and this comparison in the context of the SM is a good occasion to study the effect of this additional shift to the tadpoles and mass diagrams, thus we compute the Higgs mass in this first method both with and without using the consistent tadpole solutions. A second approach to computing $m_{h}$ with SPheno, which could potentially improve the comparison, is to use as well the same values for the top-Yukawa $y_{t}$ and electroweak gauge couplings $g_{1}, g_{2}$ as those given for each order in Table 3 of [56].

We obtain another result for $m_{h}$ with SMH [55], and although this code is made to perform Higgs mass calculations in the Standard Model to partial three-loop order, we use it here with the three-loop corrections always switched off, for the purpose of our comparison with SPheno. We use the routine calc_Mh that gives for a given loop order the value of $m_{h}$ from the inputs of the renormalisation scale $Q$, the quartic coupling $\lambda$, the top-Yukawa $y_{t}$, the Higgs VEV $v$, and the gauge couplings $g_{3}, g, g^{\prime}$, all given at scale $Q$. In order to improve the comparison, we take the same values for the inputs as used at each order in SPheno. We give in Table 1 the values we find for the Higgs mass when taking the same values of $\lambda$ as found in [56], with the two methods described above for SPheno and with SMH.

At tree level, all the values we find with SPheno and SMH obviously match as the tree-level Higgs mass only depends on $\lambda$ and $v$ which have almost the same values here, and the divergence from the value of $125.15 \mathrm{GeV}$ is solely explained by the Higgs VEV which is not the same as in [56] since they take it as an on-shell parameter, while we use the $\overline{\mathrm{MS}}$ value as described in [61]. More importantly, the loop-corrected values in the different methods also agree quite well, thanks to the improved determination of the top Yukawa coupling $y_{t}$ (including leading two-loop effects) recently implemented 
Table 1 Values of the Higgs mass at scale $Q=m_{t}$ for the values of the quartic couplings $\lambda$ found in [56] at tree level, one loop and two loops, in the two approaches we used for SPheno and with SMH. The first approach was to change only the SM parameter inputs while letting SPheno determine the top-Yukawa and electroweak gauge couplings, and the Higgs mass is computed both with and without the consistent tadpole solutions. The second method was to take the same values of

\begin{tabular}{llllll}
\hline Loop order & $\begin{array}{l}\text { Value of } \lambda \text { found } \\
\text { in [56] }\end{array}$ & $\begin{array}{l}m_{h} \text { in first approach without } \\
\text { consistent tadpole solutions }\end{array}$ & $\begin{array}{l}m_{h} \text { in first approach with } \\
\text { consistent tadpole solutions }\end{array}$ & $\begin{array}{l}m_{h} \text { in second } \\
\text { approach }\end{array}$ & $\begin{array}{l}m_{h} \text { with SMH } \\
\text { Tree level }\end{array}$ \\
\hline 0.12917 & $125.79 \mathrm{GeV}$ & $125.79 \mathrm{GeV}$ & $125.79 \mathrm{GeV}$ & $125.79 \mathrm{GeV}$ \\
One loop & 0.12774 & $125.77 \mathrm{GeV}$ & $125.77 \mathrm{GeV}$ & $125.66 \mathrm{GeV}$ & $126.10 \mathrm{GeV}$ \\
Two loops & 0.12604 & $125.11 \mathrm{GeV}$ & $125.08 \mathrm{GeV}$ & $125.10 \mathrm{GeV}$ & $125.46 \mathrm{GeV}$ \\
\hline
\end{tabular}

in SARAH [61], and at each order in perturbation theory the Higgs masses we find are less than a GeV away from $125.15 \mathrm{GeV}$. It is interesting to note that the values of $m_{h}$ found using the SPheno code generated by SARAH version 4.9 .3 - in which $y_{t}$ is only determined at one-loop order - are approximately $2-2.5 \mathrm{GeV}$ below those shown in Table 1, and hence illustrate the importance of the precise determination of the top Yukawa coupling for calculations of $m_{h}$. The small size of the difference between the values found with the couplings computed by SPheno or taken from [56] - a few tens of MeV at two loops - tend to indicate that the precision of the extraction of $y_{t}$ in SPheno is now comparable to that in [56]. Considering now the effect of the consistent tadpole solutions - which appears only in the two-loop masses - we observe a small shift of about $30 \mathrm{MeV}$ to $m_{h}$, indicating that the perturbative expansion we perform in the tadpole equation is valid for the SM. Finally, the reasons explaining the remaining deviation of our results with respect to $125.15 \mathrm{GeV}$ are the following:

1. the difference in the calculation of the Higgs VEV;

2. the two-loop electroweak corrections that are not (yet) implemented in SARAH;

3. the momentum dependence currently missing at two loops in SARAH.

The different value of the Higgs VEV is also quite certainly the main reason for the discrepancies between the values we obtain using SMH and those from [56]. I.e. it is because we use the VEV computed in SPheno in SMH, which does not correspond to the same accuracy of parameter extraction as used in [56], which would be required for a fair comparison directly between the two prior approaches: here our aim was to compare our result separately with [56] and SMH.

A further way to compare our results to those of [55] and [56] is to find for each order what value of the quartic Higgs coupling we need to obtain $m_{h}=125.15 \mathrm{GeV}$, and our results are given in Table 2 . We observe that the change of $\lambda$ between $y_{t}, g_{1}, g_{2}$ in SPheno as in [56] (and switch off the consistent tadpole routines). For SMH, the values of the input parameters - the top-Yukawa, the electroweak gauge couplings, the Higgs VEV and the strong gauge coupling - were taken from the outputs of the SPheno scans. Computations are made with SARAH-4.12.0, SPheno-4.0.3 and SMH-1.0 [55]

each order of the perturbation expansion is approximately the same in all four methods. Moreover, the value we extract at two loops with SPheno is very close to the value found in [56], only differing by $0.1 \%$.

\subsection{A detailed comparative study of SPheno and SMH results}

After this first comparison, we may now investigate in more depth the effects of the three sources of differences on the Higgs masses listed above, using SPheno and SMH. To begin with, we should consider the Higgs VEV and its calculation: in SMH, calculations are performed in the Landau gauge, while SPheno is by default set to use the Feynman gauge, and while the Higgs mass should in principle be gauge independent, its vacuum expectation value is not, hence there is an inconsistency coming from the use of a Feynman gauge $\mathrm{VEV}$ in SMH. The easiest way to correct this is to switch the SPheno calculation to the Landau gauge - we set in the code the gauge parameter $\xi$ to a very small finite value to approach the limit of the Landau gauge (the current implementation gives a numerical divergence when $\xi=0$ ) - and then to use the new value of the Landau gauge VEV in SMH. The values we find for $m_{h}^{2 \ell}$ with the two codes for the two different choices of gauge parameter and fixed values of $Q$ and $\lambda$ are given in Table 3 . The first observation that can be made from these results is that the Higgs mass shows residual dependence on the gauge $-m_{h}^{2 \ell}$ varies by about $50 \mathrm{MeV}$ between $\xi=1$ and $\xi=0.01$. This is explained mainly ${ }^{5}$ by the difference in the calculation of the $\overline{\mathrm{MS}}$ value for the electroweak VEV in SPheno between the Feynman gauge and other gauges: in the case of Feynman gauge one-loop corrections from $\delta_{V B}$ as well as two-loop corrections from $\delta_{r}$ are included which are not available in general $\xi$ (see Appendix A of [61] for details of the matching in Feynman gauge).

\footnotetext{
5 In practice, there is always an additional residual gauge dependence as the Higgs mass is computed to finite order in perturbation theory and as not all parameters used for to compute $m_{h}$ are determined to the same loop order.
} 
Table 2 Values of the Higgs quartic coupling $\lambda$ extracted from $m_{h}=125.15 \mathrm{GeV}$, at tree level, one loop and two loops. The methods we used are explained in the caption of Table 1. Values found using SARAH-4.12.0 and SMH-1.0

\begin{tabular}{llllll}
\hline Loop order & $\lambda$ found in [56] & $\begin{array}{l}\lambda \text { in first approach without } \\
\text { consistent tadpole solutions }\end{array}$ & $\begin{array}{l}\lambda \text { in first approach with con- } \\
\text { sistent tadpole solutions }\end{array}$ & $\begin{array}{l}\lambda \text { in second } \\
\text { approach }\end{array}$ \\
\hline Tree level & 0.12917 & 0.12786 & 0.12786 & 0.12786 & 0.12786 \\
One loop & 0.12774 & 0.12647 & 0.12647 & 0.12669 & 0.12580 \\
Two loops & 0.12604 & 0.12613 & 0.12619 & 0.12614 \\
\hline
\end{tabular}

On the other hand, in $\xi$ gauge, the VEV is calculated from $M_{Z}^{2, \overline{\mathrm{MS}}}=1 / 4\left(g_{1}^{2}+g_{2}^{2}\right) v^{2}=M_{Z}^{2, \text { pole }}-\Pi_{Z Z}^{T}$ where $\Pi_{Z Z}^{T}$ is the transversal self-energy of the Z-boson at one loop. What is more interesting is that the agreement between the two codes improves greatly once we use the Landau gauge in SPheno; indeed the difference in the Higgs mass results is reduced from approximately $0.4 \mathrm{GeV}$ to less than $0.05 \mathrm{GeV}$.

A second point we can study is the effect of the two-loop momentum dependence and two-loop electroweak corrections. Let us introduce the notation for calculating the pole mass via

$m_{h}^{2}=2 \lambda v^{2}+\Delta^{(1)} M_{h}^{2}\left(m_{h}^{2}\right)+\Delta^{(2)} M_{h}^{2}\left(m_{h}^{2}\right)$

where

$$
\begin{aligned}
\Delta^{(\ell)} M_{h}^{2}(s) & \equiv-\left.\frac{1}{v} \frac{\partial \Delta V^{(\ell)}}{\partial h}\right|_{h=G=G^{+}=0}+\Pi_{h h}^{(\ell)}(s) \\
& \equiv \operatorname{div}\left[\Pi_{h h}^{(\ell)}(s)\right]+\bar{\Delta}^{(\ell)} M_{h}^{2}(0)+\mathcal{O}(s),
\end{aligned}
$$

where $\operatorname{div}[f(s)]$ denotes all terms in $f(s)$ that diverge as $s \rightarrow 0$. Our SPheno code computes the one-loop corrections in any $R_{\xi}$ gauge with full momentum dependence, but the two-loop corrections are performed in a generalised effective potential approach - i.e. we keep only the divergent part of the momentum dependence (see Sect. 4 of [37] for more details). The momentum in the two-loop routines is fixed (for speed of calculation) whereas that in the one-loop routines is adjusted to solve the on-shell condition:

$$
\begin{aligned}
& s=2 \lambda v^{2}+\Delta^{(1)} M_{h}^{2}(s) \\
&+\Delta^{(2)} M_{h, \text { SPheno }}^{2}(s), \\
& \Delta^{(2)} M_{h, \text { SPheno }}^{2}(s) \equiv \operatorname{div}\left[\Pi_{h h, \text { gaugeless }}^{(2)}(s)\right] \\
&+\Delta^{(2)} M_{h, \text { gaugeless }}^{2}(0) .
\end{aligned}
$$

This begs the question of how to compare our result with SMH: ideally, we would like to extract a result from SMH which is comparable to ours. However, this is confounded by several factors:
1. It is impossible to remove or extract the electroweak contributions in $\mathrm{SMH}$, because the individual contributions in the computation diverge as the electroweak gauge couplings become zero; and the total result is not finite at vanishing external momentum.

2. To avoid the Goldstone boson catastrophe and ensure cancellation between Goldstone boson and longitudinal gauge boson diagrams, in the two-loop corrections in SMH the external momentum $s$ has been replaced by $2 \lambda v^{2}$ wherever it appears in a pre-factor (but not in the arguments of the loop functions).

3. The term

$$
\Delta^{(1)} M_{h}^{2} \supset \frac{3 \lambda}{16 \pi^{2}}\left(s^{2}-4 \lambda^{2} v^{4}\right) \frac{B(0,0)}{2 \lambda v^{2}},
$$

which is part of the one-loop correction coming from Goldstone bosons and longitudinal gauge bosons, is moved into the two-loop corrections, with the justification that on-shell $s=2 \lambda v^{2}+\Delta^{(1)} M_{h}^{2}$ so will give a contribution at two-loop order when solving for the onshell mass.

If it were not for point (2) above, it would perhaps have been possible to extract the result for the generalised effective potential approximation for the electroweak corrections. Instead, we will simply compare the results as we vary the momentum in SMH; by modifying slightly the source code, we obtain a version of SMH without the momentum dependence at two loops (but retaining the dependence at one loop). Interestingly, the result of $\mathrm{SMH}$ is finite even when $s=0$ meaning that the divergence as $s \rightarrow 0$ has been removed. It turns out that this is because of the term (3.6), which has the effect of cancelling the divergences as $s \rightarrow 0$ (even though this cancellation is fictitious). If we write $\delta^{(2)}(s)$ for the missing momentum dependence in SMH from setting the coefficients of loop functions equal to $2 \lambda v^{2}$, then we have

$$
\begin{aligned}
\Delta^{(2)} & M_{h, \mathrm{SMH}}^{2}(s) \\
= & \frac{6 \lambda}{16 \pi^{2}}\left(\Delta^{(1)} M_{h}^{2}(s)\right) B(0,0)+\Delta^{(2)} M_{h, \text { gaugeless }}^{2}(s) \\
& +\Delta^{(2)} M_{h, \text { electroweak }}^{2}(s)+\delta^{(2)}(s) \\
= & \frac{6 \lambda}{16 \pi^{2}}\left(-\frac{6 \lambda^{2} v^{2}}{16 \pi^{2}} B(0,0)+\Delta^{(1)} M_{h, \mathrm{SMH}}^{2}(0)\right) B(0,0)
\end{aligned}
$$




$$
\begin{aligned}
& +\operatorname{div}\left[\Pi_{h h, \text { gaugeless }}^{(2)}(s)+\Pi_{h h, \text { electroweak }}^{(2)}(s)+\delta^{(2)}(s)\right] \\
& +\bar{\Delta}^{(2)} M_{h, \text { gaugeless }}^{2}(0)+\bar{\Delta}^{(2)} M_{h, \text { electroweak }}^{2}(0) \\
& +\bar{\delta}^{(2)}(0)+\mathcal{O}(s) \\
& =\frac{12 \lambda}{16 \pi^{2}}\left(\bar{\Delta}^{(1)} M_{h}^{2}(0)\right)+\Delta^{(2)} M_{h, \text { gaugeless }}^{2}(0) \\
& +\Delta^{(2)} M_{h, \text { electroweak }}^{2}(0)+\bar{\delta}^{(2)}(0)+\mathcal{O}(s) .
\end{aligned}
$$

The cancellations of the divergences imply that

$$
\begin{aligned}
\operatorname{div}[ & \left.\Pi_{h h, \text { gaugeless }}^{(2)}(s)+\Pi_{h h, \text { electroweak }}^{(2)}(s)+\delta^{(2)}(s)\right] \\
\stackrel{?}{=} & \frac{1}{\left(16 \pi^{2}\right)^{2}}\left[36 \lambda^{2} v^{2} \overline{\log }^{2}(-s)-72 \lambda^{2} v^{2} \overline{\log }(-s)\right] \\
& +\frac{6 \lambda}{16 \pi^{2}}\left(\bar{\Delta}^{(1)} M_{h}^{2}(0)\right) \overline{\log }(-s),
\end{aligned}
$$

where

$$
\begin{aligned}
\left(16 \pi^{2}\right) \bar{\Delta}^{(1)} M_{h}^{2}(0) \\
\equiv\left(16 \pi^{2}\right) \Delta^{(1)} M_{h, \mathrm{SMH}}^{2}(0)-12 \lambda^{2} v^{2} \\
=-12 \lambda^{2} v^{2}+18 \lambda^{2} v^{2} \overline{\log }\left(m_{h}^{2}\right)-12 y_{t}^{2} m_{t}^{2} \overline{\log }\left(m_{t}^{2}\right) \\
\quad+\left(\frac{g_{Y}^{2}+g_{2}^{2}}{2}\right) m_{Z}^{2}\left[3 \overline{\log } m_{Z}^{2}+2\right] \\
\quad+g_{2}^{2} m_{W}^{2}\left[3 \overline{\log } m_{W}^{2}+2\right] .
\end{aligned}
$$

On the other hand, by evaluating the diagrams for the Standard Model in the gaugeless limit retaining only the top Yukawa coupling and the Higgs quartic $\lambda$ we find

Table 3 Comparison of two-loop Higgs masses calculated with the codes SPheno and SMH, for different choices of gauge in SPheno and switching on and off the two-loop momentum dependence in SMH. The renormalisation scale is fixed to $Q=173.34 \mathrm{GeV}$, and the Higgs quartic coupling is $\lambda=0.12604$ and is not varied (the idea being to illustrate the importance of the choice of scale, rather than the stability

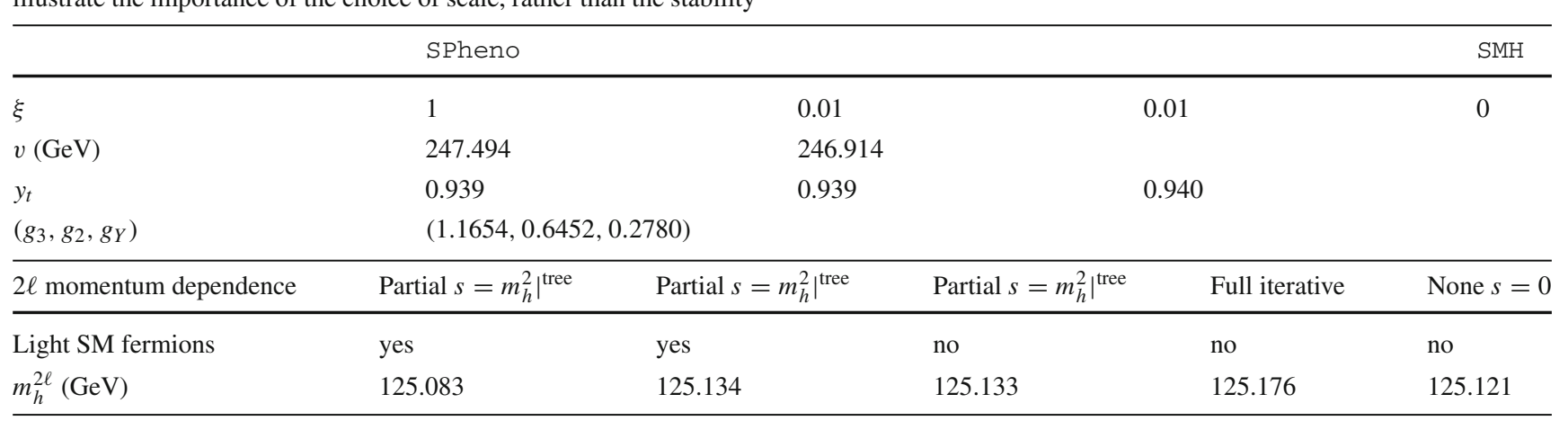

$$
\begin{aligned}
\operatorname{div} & {\left[\Pi_{h h, \text { gaugeless }}^{(2)}(s)\right] } \\
= & \frac{6 \lambda v^{2}}{\left(16 \pi^{2}\right)^{2}} \overline{\log }(-s)\left[\lambda^{2}\left(-14+18 \overline{\log }\left(m_{h}^{2}\right)+3 \overline{\log }(-s)\right)\right. \\
& \left.-2 y_{t}^{2}\left(\lambda+\left(y_{t}^{2}-\lambda\right) \overline{\log }\left(m_{t}^{2}\right)\right)\right]
\end{aligned}
$$

so we can see there are several remaining pieces that must be cancelled by $\operatorname{div}\left[\Pi_{h h \text {,electroweak }}^{(2)}(s)+\delta^{(2)}(s)\right]$. But if we set $s_{\text {fixed }}^{2}=-Q^{2}$ in our routines we should cancel the divergent part exactly, and leave us only with $\Pi_{h h \text {,gaugeless }}^{(2)}(0)$. We can then determine

$$
\begin{aligned}
\bar{\Delta}^{(2)} & M_{h, \text { electroweak }}^{2}(0)+\bar{\delta}^{(2)}(0) \\
= & \Delta^{(2)} M_{h, \text { SMH }}^{2}(0)-\Delta^{(2)} M_{h, \text { SPheno }}^{2}\left(-Q^{2}\right) \\
& -\frac{12 \lambda}{16 \pi^{2}}\left(\bar{\Delta}^{(1)} M_{h}^{2}(0)\right) .
\end{aligned}
$$

We find that this residual difference is tiny; at $Q=m_{t}=$ $173.34 \mathrm{GeV}$ with $\lambda=0.12604, y_{t}=0.9345, v=247.07$ $\mathrm{GeV}$ and the gauge couplings $\left(g_{3}, g_{2}, g_{Y}\right)$ $=(1.1654,0.6442,0.2782)$ we have

$$
\begin{aligned}
\bar{\Delta}^{(2)} M_{h, \text { electroweak }}^{2}(0)+\bar{\delta}^{(2)}(0) & \simeq-0.03(\mathrm{GeV})^{2} \\
& =-0.0002 \% m_{h}^{2} !
\end{aligned}
$$

This corresponds to a tiny value of the electroweak corrections; a similar observation was made in [56].

Finally, we compare the more physically meaningful differences between the codes when we take $s=\left.m_{h}^{2}\right|^{\text {tree }}$ in our routines. The values of the Higgs mass computed with SPheno after turning off the light SM fermion contributions and with the modified version of SMH is given in Table 3, and strikingly they only differ by $40 \mathrm{MeV}$ when we include the momentum dependence in $\mathrm{SMH}$ - in other words, for

of the result). All other inputs for SMH are taken to the same values as in SPheno. In SPheno the only two-loop momentum dependence is from pseudo-scalar diagrams and only a generalised effective potential approach (see main text) with $s=\left.m_{h}^{2}\right|^{\text {tree }}$, while in SMH the full two-loop dependence is implemented and is used to find $m_{h}$ iteratively 


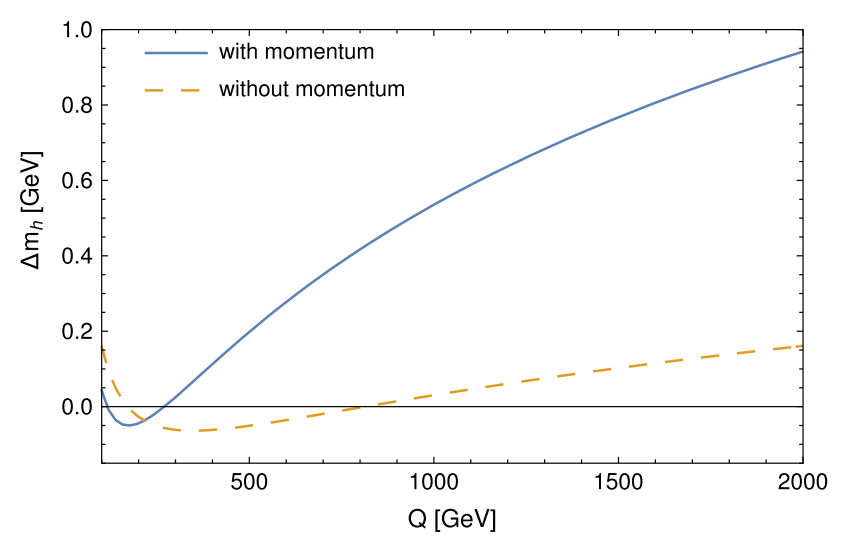

Fig. 1 Difference between the two-loop Higgs mass computed by SMH and SPheno- $\left(m_{h}^{2 \ell}\right)^{\mathrm{SPheno}}-\left(m_{h}^{2 \ell}\right)^{\mathrm{SMH}}-$ as a function of the renormalisation scale $Q$, with (blue curve) and without (orange dashed curve) the momentum dependence at two loops in SMH. The Higgs quartic coupling is here $\lambda=0.12604$. In SPheno the contributions of the light SM fermions are turned off and the external momentum in the two-loop routines is set to $s=\left.m_{h}^{2}\right|^{\text {tree }}$

$Q=173.34 \mathrm{GeV}$, the momentum dependence and electroweak corrections amount to only $0.03 \%$ of $m_{h}$. We further examine the importance of both the momentum dependence and the EW corrections by varying now the renormalisation scale at which we compute the Higgs mass: for this purpose, Fig. 1 shows the difference of the two-loop masses between the two codes - more precisely $\left(m_{h}^{2 \ell}\right)^{\text {SPheno }}-\left(m_{h}^{2 \ell}\right)^{\text {SMH }}-$ with and without momentum, as a function of the renormalisation scale $Q$ (where the $\overline{\mathrm{MS}}$ parameters are extracted by SPheno at each value while keeping $\lambda$ fixed rather than evolving the parameters: the idea is to show the importance of the choice of scale rather than the stability of the computation). While for large scales the two-loop momentum effects may become large ( $1 \mathrm{GeV}$ or more), the electroweak corrections represent at most $0.2 \mathrm{GeV}$ and even vanish for a scale close to the $\overline{\mathrm{MS}}$ top mass.

\subsection{Momentum dependence}

Implementing the solution to the Goldstone boson catastrophe in SARAH has required the insertion of external momentum in infra-red divergent loop integrals, and thus we should also investigate the impact of the momentum $s=-p^{2}$ on the Higgs mass calculation in SPheno. In practise, we have set for the majority of scans the external momentum for the twoloop calculations to be equal to $m_{h}^{2}$ tree but we will now vary the momentum to study its impact on $m_{h}$. Table 4 shows the shift to the two-loop Higgs mass - with respect to the value computed with $s=(125 \mathrm{GeV})^{2}-$ for external momentum in loops equal to $s=\alpha \times(125 \mathrm{GeV})^{2}$, where $\alpha$ ranges from $10^{-6}$ to $10^{6}$ and for $\lambda=0.126$ and $\lambda=0.130$. For all values of the external momentum considered here, the variation of the Higgs mass remains small: at most they become of order $\sim 0.13 \times 1 \mathrm{GeV}$ for $\alpha=10^{-6}$ (i.e. $\sqrt{s}=0.125 \mathrm{GeV}$ ), and while this effect is noticeable, it is far from the divergences that could have been feared when approaching the limit of $s \rightarrow 0$. All in all, although pole masses - as we compute here - are in principle found as the zero of the inverse propagator, that has to be found iteratively as the self-energy contains momentum dependence, we see from the minute effects of momentum in the range $\alpha \in[1 / 2,100]$, relevant for scalar masses, that we will not require an iterative solution and that simply taking $s=(125 \mathrm{GeV})^{2}$ in the loop diagrams with pseudo-scalars will be a satisfactory approximation. In particular, changing $s$ between $\left.m_{h}^{2}\right|^{\text {tree }}$ and $125 \mathrm{GeV}$ causes a difference in $m_{h}^{2 \ell}$ of less than an $\mathrm{MeV}$.

We emphasise, however, that the effect of momentum on Goldstone boson mass diagrams discussed here is only a subset of the general momentum dependence of the two-loop masses, which should in principle be taken into account, as seen in the previous sections.

\section{The NMSSM}

As a second check of our new solution, and demonstration of its importance, we shall compare the results for the three different options to solve the Goldstone Boson Catastrophe in the example of the next-to-minimal supersymmetric standard model (NMSSM) - see [62] and the references therein for a detailed description of the model. Indeed, the NMSSM is the first supersymmetric model for which the problems at certain points in the parameter space were found in earlier versions of SARAH. Here we shall show that this is avoided, and have a preliminary look at the impact of the "consistent tadpole solutions".

We start with a test point defined by the following input parameters: ${ }^{6}$

$$
\begin{aligned}
\lambda & =0.7, \quad \kappa=0.25, \quad A_{\lambda}=1350 \mathrm{GeV}, \\
A_{\kappa} & =-500 \mathrm{GeV}, \quad \mu_{\mathrm{eff}}=600 \mathrm{GeV}, \\
M_{1} & =M_{2}=1000 \mathrm{GeV}, \quad M_{3}=2000 \mathrm{GeV}, \\
T_{u, 33} & =1500 \mathrm{GeV}, \quad m_{\tilde{u}, 33}=1000 \mathrm{GeV},
\end{aligned}
$$

and all other soft-masses set to $2 \mathrm{TeV}$. The Higgs masses for the following calculations are given in Table 5:

1. D-terms turned off in mass matrices but retained in tadpole solutions (as in previous versions of SARAH), labelled " $\mathrm{D}$ " in the table.

2. Regulator masses with $R=10^{-5}-10^{-1}$.

\footnotetext{
${ }^{6}$ Note, in this section we use $\lambda$, which is in all other sections the quartic Higgs coupling from the SM, for the superpotential coupling $\hat{S} \hat{H}_{d} \hat{H}_{u}$ as usually done in the NMSSM.
} 
Table 4 Shift in GeV of the two-loop Higgs mass in the Standard Model - computed with SPheno and with respect to the value obtained for $p=125 \mathrm{GeV}$ - for different values of the quartic coupling $\lambda$, and of the incoming momentum $s$ in the two-loop routines

\begin{tabular}{|c|c|c|c|c|c|c|c|c|c|}
\hline \multirow[t]{2}{*}{ Value of $\lambda$} & \multicolumn{9}{|c|}{ Shift to the two-loop Higgs mass for the values of the momentum $s=\alpha \times(125 \mathrm{GeV})^{2}$, in GeV } \\
\hline & $\alpha=10^{-6}$ & $\alpha=10^{-4}$ & $\alpha=10^{-2}$ & $\alpha=1 / 2$ & $\alpha=1$ & $\alpha=2$ & $\alpha=100$ & $\alpha=10^{4}$ & $\alpha=10^{6}$ \\
\hline 0.126 & 0.1210 & 0.0655 & 0.0252 & 0.0028 & 0.0 & -0.0025 & -0.0100 & -0.0048 & 0.0155 \\
\hline 0.130 & 0.1302 & 0.0704 & 0.0270 & 0.0030 & 0.0 & -0.0026 & -0.0106 & -0.0048 & 0.0560 \\
\hline
\end{tabular}

Table 5 The Higgs masses in the NMSSM (in GeV) for the parameter point defined by Eq. (4.1) for different choices for the two-loop corrections

\begin{tabular}{rrrrrrrrr}
\hline & \multicolumn{1}{c}{$\mathrm{D}$} & $R=10^{-5}$ & $R=10^{-4}$ & $R=10^{-3}$ & $R=10^{-2}$ & $R=10^{-1}$ & \multicolumn{1}{c}{ OS } & OS+Tad \\
\hline$h_{1}$ & 129.58 & 65.27 & 124.63 & 129.07 & 129.82 & 130.58 & 129.70 & 129.97 \\
$h_{2}$ & 315.64 & 312.84 & 315.39 & 315.59 & 315.55 & 315.67 & 315.09 & 315.60 \\
$h_{3}$ & 1632.28 & 1627.55 & 1631.77 & 1632.36 & 1632.63 & 1632.81 & 1632.51 & 1633.39 \\
$A_{1}$ & 582.02 & 582.61 & 582.31 & 582.02 & 581.74 & 581.63 & 580.94 & 581.23 \\
$A_{2}$ & 1631.98 & 1630.38 & 1631.15 & 1631.88 & 1632.43 & 1632.59 & 1632.04 & 1632.60 \\
\hline
\end{tabular}

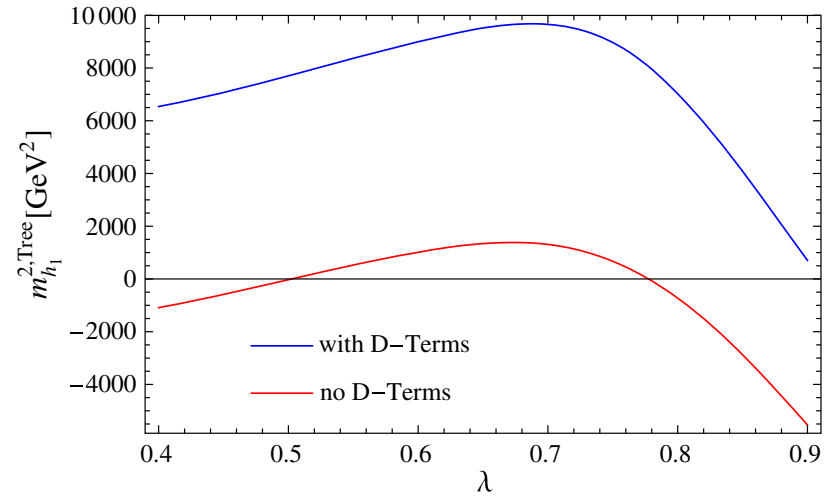

Fig. 2 The lightest scalar mass squared for the parameter point defined by Eq. (4.2) when calculating with and without D-term contributions

3. Goldstones set on-shell, with and without consistent tadpole solutions, labelled OS and OS+Tad, respectively.

We see from this table that there is an agreement in the light Higgs mass of about $0.4 \mathrm{GeV}$ between all the calculations if $R$ is chosen to be about $10^{-2}$.

While the new "on-shell" solution of the Goldstone boson catastrophe is optimal, between introducing a regulator $R$ and the previous approach with neglected D-terms in the scalar mass matrix, the latter is preferred because one does not need to check for a suitable choice of $R$ to stabilise the results. However, we can now consider parameter points where the old method fails. This is shown for the point defined by

$$
\begin{aligned}
\kappa & =0.6, \quad A_{\lambda}=200 \mathrm{GeV}, \quad A_{\kappa}=-200 \mathrm{GeV}, \\
\mu_{\mathrm{eff}} & =150 \mathrm{GeV}, \\
M_{1} & =M_{2}=1000 \mathrm{GeV}, \quad M_{3}=2000 \mathrm{GeV},
\end{aligned}
$$

and all scalar soft-masses set to $2 \mathrm{TeV}$. The lightest scalar tree-level mass with and without the D-terms as a function

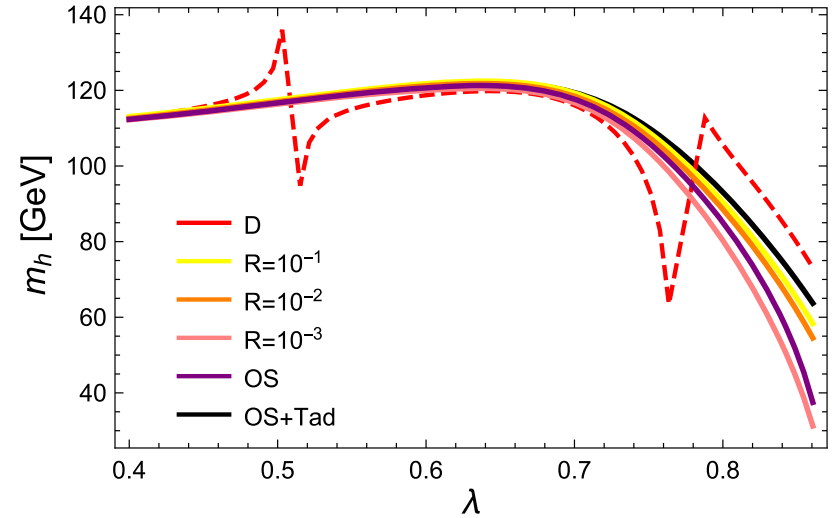

Fig. 3 The lightest Higgs mass at the two-loop level for the parameter point defined by Eq. (4.2) for different methods to regulate the two-loop corrections

of $\lambda$ is shown in Fig. 2. One can see that for $\lambda \simeq 0.5,0.8$, the lightest scalar becomes massless in the limit of vanishing D-terms. Thus, for these values, divergences in the two-loop corrections can be expected which are this time not associated with the Goldstone but with the lightest CP-even state. We show the lightest Higgs mass in Fig. 3 as a function of $\lambda$ for different methods to regulate the two-loop corrections. Obviously, the approach of neglecting electroweak D-terms fails for values of $\lambda$ at which the masses entering the loop calculations become very light. However, for very large values of $\lambda$ which are away from the poles, the agreement with the other calculations is also rather poor. In contrast, over the entire range of $\lambda$ we see good agreement between the methods using regulator masses, if $R=10^{-2}$ or $10^{-3}$ is chosen, and the method of treating the Goldstones on-shell. It is interesting that for these values of $R$ the minimum mass is $\sqrt{R} \times M_{\text {SUSY }} \simeq 100 \mathrm{GeV}$, i.e. logarithmic contributions involving the light scalars are being excised. 
We note that the corrections from the consistent tadpole solution are small until $\lambda$ becomes large, at which point we see significant deviations. However, as $\lambda$ approaches 0.9 we see from Fig. 2 that the tree-level lightest Higgs mass approaches zero, so we expect our perturbative calculation of the "consistent tadpole solution" to break down and become unreliable.

\section{Split SUSY}

In Split SUSY scenarios [63-68], the SUSY scalars are much heavier than the gauginos and Higgsinos. Consequently, these models should be studied in an effective approach where all SUSY scalars are integrated out at some matching scale. The Lagrangian below this scale is given by

$$
\begin{aligned}
\mathcal{L}= & \mathcal{L}_{\mathrm{SM}}-\left(\frac{1}{2} M_{3} \tilde{g}^{\alpha} \tilde{g}^{\alpha}+\frac{1}{2} M_{2} \tilde{W}^{a} \tilde{W}^{a}\right. \\
& \left.+\frac{1}{2} M_{B} \tilde{B} \tilde{B}+\mu \tilde{H}_{u}^{T} \epsilon \tilde{H}_{d}+\text { h.c. }\right) \\
- & {\left[\frac{1}{\sqrt{2}} H^{\dagger}\left(\tilde{g}_{2 u} \sigma^{a} \tilde{W}^{a}+\tilde{g}_{1 u} \tilde{B}\right) \tilde{H}_{u}\right.} \\
& \left.+\frac{1}{\sqrt{2}} H^{T} \epsilon\left(-\tilde{g}_{2 d} \sigma^{a} \tilde{W}^{a}+\tilde{g}_{1 d} \tilde{B}\right) \tilde{H}_{d}+\text { h.c. }\right]
\end{aligned}
$$

where $\mathcal{L}_{\mathrm{SM}}$ is the Standard Model Lagrangian with Higgs potential (3.1). Because of the matching between the effective, non-supersymmetric model and the MSSM, the quartic Higgs coupling $\lambda$ as well as the new Yukawa-like interactions $\tilde{g}_{(1,2)(u, d)}$ are not free parameters but fixed by the matching conditions at the scale $M_{M}$. At tree level, the following relations hold:

$$
\begin{aligned}
\tilde{g}_{2 u}\left(M_{M}\right) & =g_{2}\left(M_{M}\right) \sin \beta, \\
\tilde{g}_{2 d}\left(M_{M}\right) & =g_{2}\left(M_{M}\right) \cos \beta, \\
\tilde{g}_{1 u}\left(M_{M}\right) & =\sqrt{\frac{3}{5}} g_{1}\left(M_{M}\right) \sin \beta, \\
\tilde{g}_{1 d}\left(M_{M}\right) & =\sqrt{\frac{3}{5}} g_{1}\left(M_{M}\right) \cos \beta, \\
\lambda\left(M_{M}\right) & =\frac{1}{8}\left(g_{1}^{2}\left(M_{M}\right)+g_{2}^{2}\left(M_{M}\right)\right) \cos ^{2} 2 \beta .
\end{aligned}
$$

Here, $g_{1}$ and $g_{2}$ are the running gauge couplings of $U(1)_{Y} \times$ $S U(2)_{L}$ and $\beta$ is defined as the mixing angle of the two Higgs doublets in the MSSM (in contrast to the definition in the MSSM as a ratio of expectation values). There are important higher-order corrections to the matching conditions which are necessary to have a precise prediction for the Higgs mass at the low scale. In particular $\lambda$ has been calculated including the two-loop SUSY corrections [6971]. The numerical value of these corrections depends on the many SUSY parameters at the matching scale; however, a commonly taken useful approximation is to give the scalars a common mass $M_{M}$, in which case the corrections can be given in terms of just this scale and the squark mixing. Moreover, in strict split SUSY where the fermion masses are protected by an R-symmetry (or another symmetry in Fake Split SUSY [72,73]) near the electroweak or $\mathrm{TeV}$ scale and well below $M_{M}$, the squark mixing must by very small. In which case, the leading corrections to the Higgs quartic coupling are purely electroweak at one loop, and at two loops contain no logarithmic terms - meaning that they are very small (in particular since the strong gauge and top Yukawa couplings run to small values at higher scales), so using the tree-level relationship above can be good enough.

Below the scale $M_{M}$, we must run to the scale of the fermion masses, before also integrating them out, and then running to the electroweak scale in the Standard Model. In some previous approaches, the running was performed all the way down to the electroweak scale, before calculating the Higgs mass in the full Lagrangian (5.1); however, it was found in [72] that in this approach it is necessary to include the three-loop leading logarithm involving the gluino mass to obtain good agreement between the two results - this is automatically resummed by the renormalisation group running in the former approach. In either case, the full contribution of the gauginos and Higgsinos to the matching conditions is only known in the literature to one-loop order [69].

Hence in this section we are interested in the effect of the two-loop corrections to the Higgs mass stemming from the $\tilde{g}_{(1,2)(u, d)}$ couplings which have not been studied in the literature before. They are expected to be small since they originate from electroweak interactions at the matching scale (and so, admittedly, one could argue that we should neglect them in the gaugeless limit). We shall not discuss the absolute value of the Higgs mass, for which we would need to include all higher-order corrections to the matching that have been calculated elsewhere, but only on the impact of the new two-loop corrections. The overall size of these corrections is rather insensitive to the exact matching conditions and we are using the above tree-level relations; but as we noted earlier, these should be a particularly good approximation for larger matching scales.

We make in addition the simplifying assumption that at $M_{M}$ the SUSY fermions are degenerate, i.e.

$\mu\left(M_{M}\right)=M_{1}\left(M_{M}\right)=M_{2}\left(M_{M}\right)=M_{3}\left(M_{M}\right) \equiv M_{F}$

and thus we are left with three free parameters:

$M_{F}, \quad M_{M}, \quad \tan \beta$. 

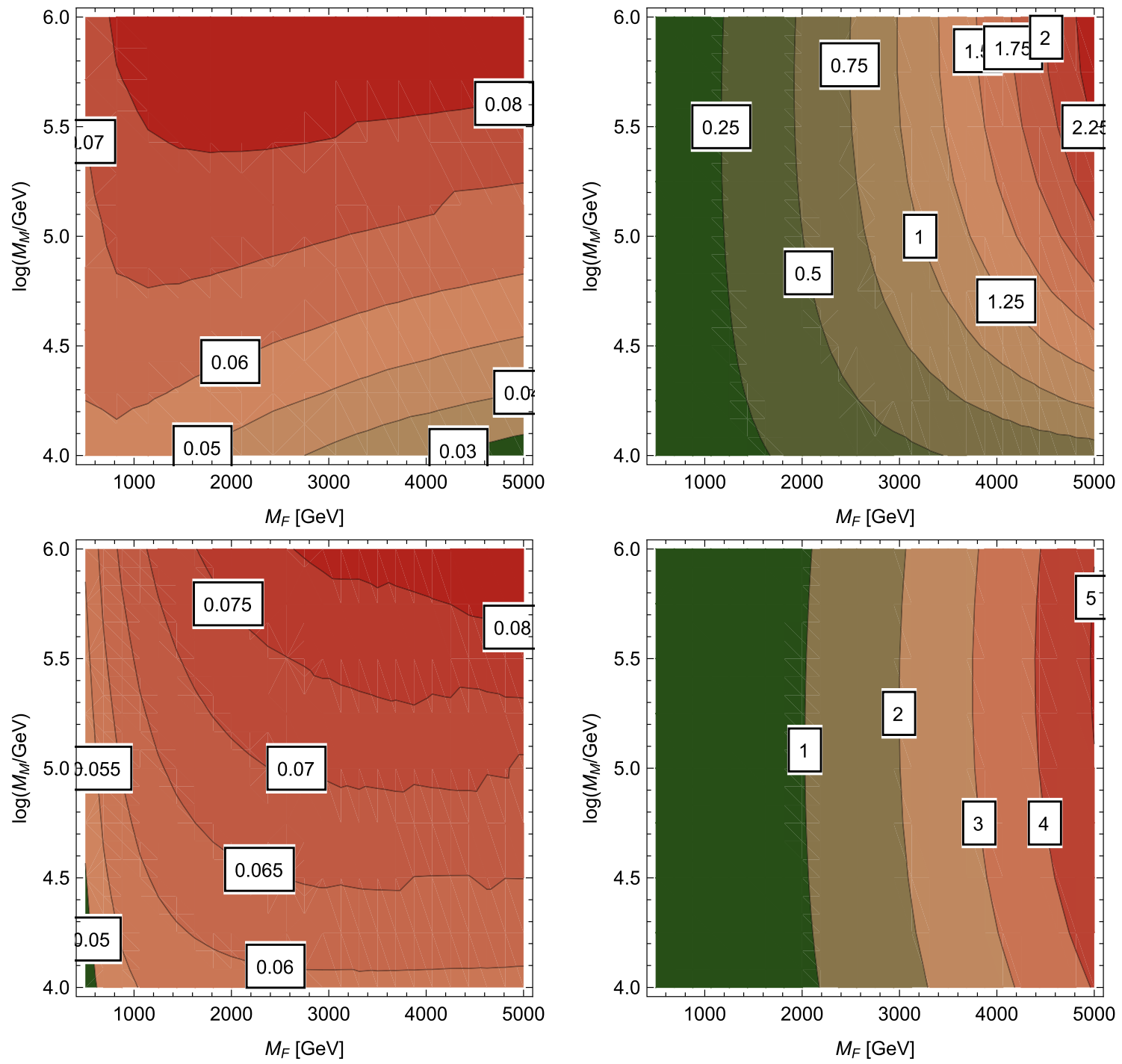

Fig. 4 The change in the Higgs mass in GeV due to the two-loop corrections involving the new Yukawa-like interactions $\tilde{g}_{(1,2)(u, d)}$. On the top, we used $\tan \beta=1$ at $M_{M}$ and on the bottom $\tan \beta=10$. The left plots are with the consistent tadpole solutions, the right ones without

SARAH uses two-loop RGEs for the running between $M_{M}$ and the renormalisation scale $Q$ which we set to $M_{F}$, which as mentioned above is necessary to avoid large logarithmic contributions from the gluino. The size of the two-loop corrections proportional to the $\tilde{g}_{(1,2)(u, d)}$ couplings in the $\left(M_{M}, M_{F}\right)$ plane is shown in Fig. 4 for $\tan \beta=1$ and 10 for a calculation with and without the consistent tadpole solutions explained in Sect. 2.2. We show here results for $M_{F}$ up to $5 \mathrm{TeV}$. In order not to increase the theoretical uncertainty in the presence of new fermions in the multi- $\mathrm{TeV}$ range, we made use of the functionality in SARAH to perform the Higgs mass calculation in the effective SM [61]. For this purpose, a second matching is performed to extract $\lambda$ at the renormalisation scale $Q$. The imposed matching condition is
$m_{h}^{\mathrm{SM}}\left(M_{F}\right) \equiv m_{h}^{\mathrm{Split}}\left(M_{F}\right)$

i.e. we perform a matching of the Higgs pole masses as suggested in [54], from which an effective $\lambda$ is derived. $\lambda$ is then evolved to $m_{t}$ using three-loop RGEs of the SM. At $m_{t}$ the Higgs mass is calculated within the SM at the two-loop level. The additional loop corrections discussed here enter the calculation of $m_{h}^{\text {Split }}\left(M_{F}\right)$, i.e. the calculation of $\lambda^{\mathrm{SM}}\left(M_{F}\right)$.

We see that the additional corrections for SUSY fermions are always well below $1 \mathrm{GeV}$ once the consistent solution to the tadpole equations are included. However, if those are not used, the misleading impression of sizeable corrections of a few $\mathrm{GeV}$ is given; it would be interesting to investigate this phenomenon further. 


\section{Two-Higgs-Doublet Model}

The scalar potential of the CP-conserving 2HDM is defined in terms of scalar $S U(2)_{L}$ doublets in a basis $\left\{\Phi_{1}, \Phi_{2}\right\}-$ sometimes called the $\mathbb{Z}_{2}$ basis - as

$$
\begin{aligned}
V^{(0)}= & m_{11}^{2}\left(\Phi_{1}^{\dagger} \cdot \Phi_{1}\right)+m_{22}^{2}\left(\Phi_{2}^{\dagger} \cdot \Phi_{2}\right) \\
& +m_{12}^{2}\left(\Phi_{1}^{\dagger} \cdot \Phi_{2}+\Phi_{2}^{\dagger} \cdot \Phi_{1}\right) \\
& +\lambda_{1}\left(\Phi_{1}^{\dagger} \cdot \Phi_{1}\right)^{2}+\lambda_{2}\left(\Phi_{2}^{\dagger} \cdot \Phi_{2}\right)^{2} \\
& +\lambda_{3}\left(\Phi_{1}^{\dagger} \cdot \Phi_{1}\right)\left(\Phi_{2}^{\dagger} \cdot \Phi_{2}\right) \\
& +\lambda_{4}\left(\Phi_{1}^{\dagger} \cdot \Phi_{2}\right)\left(\Phi_{2}^{\dagger} \cdot \Phi_{1}\right) \\
& +\frac{1}{2} \lambda_{5}\left[\left(\Phi_{1}^{\dagger} \cdot \Phi_{2}\right)^{2}+\left(\Phi_{2}^{\dagger} \cdot \Phi_{1}\right)^{2}\right] .
\end{aligned}
$$

One or both doublet(s) $\Phi_{1}$ and $\Phi_{2}$ may acquire VEVs if $m_{i j}^{2}$ has one or two negative eigenvalues, and we write the doublets and their VEVs as

$\Phi_{i}=\left(\begin{array}{c}\Phi_{i}^{+} \\ \Phi_{i}^{0}\end{array}\right) \quad$ and $\quad\left\langle\Phi_{i}\right\rangle=\frac{1}{\sqrt{2}}\left(\begin{array}{c}0 \\ v_{i}\end{array}\right), \quad$ for $i=1,2$

We then define the angle $\beta$ through the usual relation

$\tan \beta=\frac{v_{2}}{v_{1}} \Leftrightarrow\left\{\begin{array}{l}v_{1}=v \cos \beta \\ v_{2}=v \sin \beta\end{array}\right.$

where $v$ is defined by $v^{2}=v_{1}^{2}+v_{2}^{2}$. The 2HDM hence has seven free parameters, which are

$\lambda_{i}($ for $i \in\{1,2,3,4,5\}) ; \quad m_{12}^{2} ; \quad \tan \beta$.

It is often more convenient to work in another basis - the so-called Higgs basis $\left\{H_{1}, H_{2}\right\}$ - where the neutral component of the doublet $H_{1}$ is aligned in field space with the total $\operatorname{VEV} v$, with a rotation of angle $\beta$

$\left\{\begin{array}{l}\Phi_{1}=H_{1} c_{\beta}-H_{2} s_{\beta}, \\ \Phi_{2}=H_{1} s_{\beta}+H_{2} c_{\beta},\end{array} \Leftrightarrow\left\{\begin{array}{l}H_{1}=\Phi_{1} c_{\beta}+H_{2} s_{\beta}, \\ H_{2}=-\Phi_{1} s_{\beta}+\Phi_{2} c_{\beta} .\end{array}\right.\right.$

We choose to write these two new doublets as

$$
H_{1}=\left(\begin{array}{c}
H_{1}^{+} \\
\frac{1}{\sqrt{2}}\left(v+H_{1}^{0}\right)
\end{array}\right), \quad H_{2}=\left(\begin{array}{c}
H_{2}^{+} \\
\frac{1}{\sqrt{2}} H_{2}^{0}
\end{array}\right)
$$

In this new basis, following the notation of [74], the potential can be written as

$$
\begin{aligned}
V^{(0)}= & Y_{1}\left(H_{1}^{\dagger} \cdot H_{1}\right)+Y_{2}\left(H_{2}^{\dagger} \cdot H_{2}\right) \\
& +Y_{3}\left(H_{1}^{\dagger} \cdot H_{2}+H_{2}^{\dagger} \cdot H_{1}\right) \\
& +\frac{Z_{1}}{2}\left(H_{1}^{\dagger} \cdot H_{1}\right)^{2}+\frac{Z_{2}}{2}\left(H_{2}^{\dagger} \cdot H_{2}\right)^{2} \\
& +Z_{3}\left(H_{1}^{\dagger} \cdot H_{1}\right)\left(H_{2}^{\dagger} \cdot H_{2}\right) \\
& +Z_{4}\left(H_{1}^{\dagger} \cdot H_{2}\right)\left(H_{2}^{\dagger} \cdot H_{1}\right) \\
& +\frac{1}{2} Z_{5}\left[\left(H_{1}^{\dagger} \cdot H_{2}\right)^{2}+\left(H_{2}^{\dagger} \cdot H_{1}\right)^{2}\right] \\
& +\left[Z_{6}\left(H_{1}^{\dagger} \cdot H_{1}\right)+Z_{7}\left(H_{2}^{\dagger} \cdot H_{2}\right)\right] \\
& \times\left[H_{1}^{\dagger} \cdot H_{2}+H_{2}^{\dagger} \cdot H_{1}\right] .
\end{aligned}
$$

The CP-even physical states are eigenstates of the mass matrix

$$
\mathcal{M}_{H}^{2}=\left(\begin{array}{cc}
Z_{1} v^{2} & Z_{6} v^{2} \\
Z_{6} v^{2} & m_{A}^{2}+Z_{5} v^{2}
\end{array}\right)
$$

where $m_{A}^{2}=-\frac{2 m_{12}^{2}}{s_{2 \beta}}-\lambda_{5} v^{2}$,

which is diagonalised with an angle $\alpha$, and they are given by

$$
\left\{\begin{array}{c}
h=\left(\sqrt{2} \mathfrak{R e}\left(\Phi_{1}^{0}\right)-v_{1}\right) s_{\alpha}+\left(\sqrt{2} \mathfrak{R e}\left(\Phi_{2}^{0}\right)-v_{2}\right) c_{\alpha} \\
=\mathfrak{R e}\left(H_{1}^{0}\right) s_{\beta-\alpha}+\mathfrak{R e}\left(H_{2}^{0}\right) c_{\beta-\alpha}, \\
H=\left(\sqrt{2} \mathfrak{R e}\left(\Phi_{1}^{0}\right)-v_{1}\right) c_{\alpha}-\left(\sqrt{2} \mathfrak{R e}\left(\Phi_{2}^{0}\right)-v_{2}\right) s_{\alpha} \\
=\mathfrak{R e}\left(H_{1}^{0}\right) c_{\beta-\alpha}-\mathfrak{R e}\left(H_{2}^{0}\right) s_{\beta-\alpha}
\end{array}\right.
$$

The alignment limit is defined as the limit in which the neutral components of the Higgs-basis doublets are also mass eigenstates, or in other words, the limit in which one of the $\mathrm{CP}$-even neutral scalar mass eigenstates is aligned with the VEV $v$. From Eq. (6.10) we see that this can be realised in two ways:

1. $s_{\beta-\alpha}=0$ in which $H$ carries the VEV and is identified with the SM-like Higgs.

2. $c_{\beta-\alpha}=0$ which means that $h$ is the SM-like Higgs.

We do not make any assumption on the size of the masses of the different scalars i.e. we do not suppose that we are in the decoupling limit as well. Consequently, at tree level we only require $Z_{6} v^{2} \rightarrow 0$, and hence with the expression of $Z_{6}$ derived in [74], we have

$Z_{6} \equiv-s_{2 \beta}\left[\lambda_{1} c_{\beta}^{2}-\lambda_{2} s_{\beta}^{2}-\frac{1}{2} \lambda_{345} c_{2 \beta}\right]=0$ 
where $\lambda_{345} \equiv \lambda_{3}+\lambda_{4}+\lambda_{5}$. The simplest, and $\tan \beta$ independent, way to fulfil this condition is to have

$\lambda_{1}=\lambda_{2}=\frac{1}{2} \lambda_{345}$,

which we will use in the following to constrain tree-level alignment. Also, we will require that the SM-like Higgs be the lightest mass eigenstate $h$ (case (2) above), by ensuring that

$Z_{1} v^{2}<m_{A}^{2}+Z_{5} v^{2}$

This implies that $c_{\beta-\alpha}=0$, and thus, with the conventional choice that $\beta \in\left[0, \frac{\pi}{2}\right]$ and $|\alpha| \leq \frac{\pi}{2}$, we have

$\beta-\alpha=\frac{\pi}{2} \Rightarrow \alpha \in\left[-\frac{\pi}{2}, 0\right]$.

The constraint for tree-level alignment given in Eq. (6.12) reduces the number of free parameters of the model from seven to five, as two of the quartic couplings (e.g. $\lambda_{2}$ and $\lambda_{3}$ ) can be found as a function of the three other ones.

For most scans and figures presented below, we worked in the type-I $2 \mathrm{HDM}$ if not indicated otherwise. However as the difference with type-II comes from the couplings of the scalars to the down-type quarks and to the leptons which are light and give much smaller contributions to the lightest Higgs mass than the top quark, we do not expect large effects on our results (even for large $\tan \beta$, since the contributions typically involve the quark masses rather than just the couplings).

\subsection{Renormalisation scale dependence of the Higgs mass computed with SPheno}

The masses computed by SPheno are pole masses, which should in principle not depend on the renormalisation scale at which they are computed. Evaluating the variation of the masses with the scale $Q$ hence provides a consistency check of our results and an estimate of the theoretical uncertainty as the variation of the two-loop masses with $Q$ corresponds roughly to the three-loop corrections. For this purpose, we have tuned the $\lambda_{i}$ couplings to ensure a two-loop Higgs mass of $125.09 \mathrm{GeV}$, at scale $Q=160 \mathrm{GeV}$, together with tree-level alignment and find the following values (using HiggsBounds we have verified that this point in parameter space is not excluded by the current experimental constraints):

$$
\begin{aligned}
\lambda_{1} & =\lambda_{2}=0.0911, \quad \lambda_{3}=0.3322, \\
\lambda_{4} & =0.8000, \quad \lambda_{5}=-0.9500, \\
m_{12}^{2} & =-50000 \mathrm{GeV}^{2}, \quad \tan \beta=50 .
\end{aligned}
$$

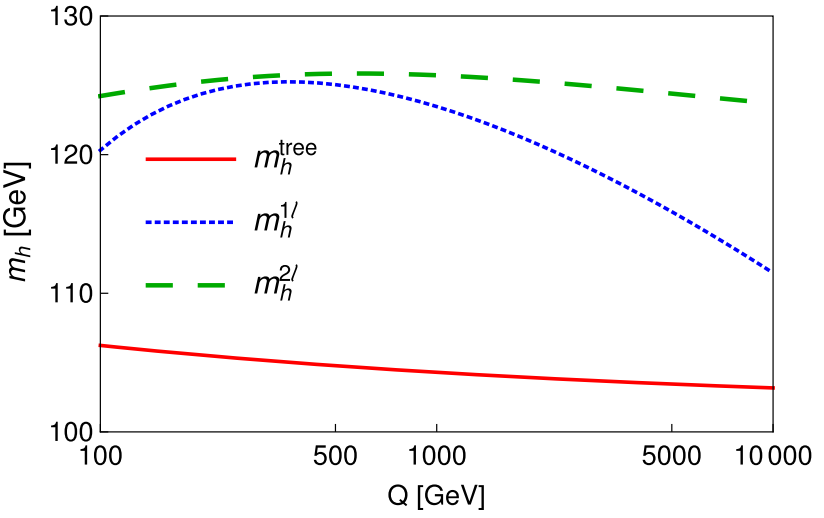

Fig. 5 Lightest Higgs mass $m_{h}^{2}$ as a function of the renormalisation scale $Q$, considering only the running of SM parameters. Red curve: tree level; blue dot-dashed curve: one-loop order; green dashed curve: two-loop order

At first we consider that these inputs are then given to SPheno as the value of the couplings at a scale $Q$ that we vary in the range $[100 \mathrm{GeV}, 10,000 \mathrm{GeV}]$, and we only consider the running of SM parameters; we find the results shown in Fig. 5 for the tree-level, one-loop and two-loop Higgs mass $m_{h}$. Since phenomenological analyses typically supply the quartic couplings without reference to a higher-energy theory or the scale where they are determined, this plot shows the importance of the choice of that scale. We have verified that the renormalisation scale dependence of $\left.m_{h}\right|^{\text {tree }}$ is entirely due to the scale dependence of the Higgs VEV $v$, as the running of the quartic couplings is for the moment not applied. The renormalisation scale $Q$ is seen to have only a limited effect on the two-loop value of $m_{h}$ which varies of about $2 \mathrm{GeV}$ on the range of scales considered here, while the one-loop result varies by about $15 \mathrm{GeV}$. Since the twoloop curve is so flat, this shows that most of the variation in the calculation of the Higgs mass for the chosen quartics must come from variation of the Standard Model parameters, and that a two-loop calculation (rather than one-loop) is necessary not just for precision but also to ensure scale stability.

Using two-loop RGEs implemented in SARAH/SPheno, we can also include the evolution of the 2HDM parameters to obtain a more complete scale dependence of the masses, as shown in Fig. 6. Once more, the two-loop value of Higgs mass depends less on the renormalisation scale than the tree-level or one-loop values. This weaker dependence of the two-loop Higgs mass on $Q$, compared with the one-loop mass, even for choices of parameters that give large loop corrections is a first verification of the validity of our new two-loop routines. In the following we will therefore work at a fixed scale $Q=m_{t}$, confident that the results will be for the most part independent of this choice. 


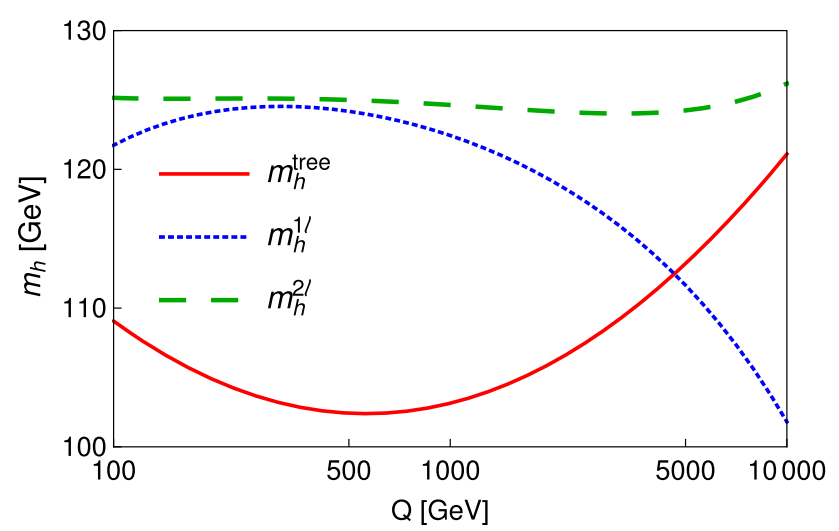

Fig. 6 Lightest Higgs mass $m_{h}^{2}$ as a function of the renormalisation scale $Q$, taking into account the running of all parameters with the RGEs included in SPheno. Red curve: tree level; Blue dot-dashed curve: one-loop order; Green dashed curve: two-loop order

\subsection{Quantum corrections to the alignment limit}

The relations defining the alignment limit in the beginning of this section are only valid at tree level and we expect them to receive corrections at one- and two-loop order, and in this section we will discuss the importance of these effects on the mixing angle of the neutral CP-even scalars $\alpha$.

Scanning over the different free couplings of the model - $m_{12}^{2}$ and $\lambda_{i}(i \in\{3,4,5\})$ - we compare the values of the CP-even Higgs mixing angle $\alpha$ at tree level, one-loop and two-loop order, as shown in Fig. 7, and as expected, loop corrections cause deviations from the tree-level relation $t_{\alpha}=-1 / t_{\beta} \Leftrightarrow c_{\beta-\alpha}=0$. The observations we can make from these plots are the following:

1. in the ranges of the parameters that we considered, the effect of loop corrections on the value of $\alpha$ is small, at most of the order of $1 \%$;

2. the one-loop corrections to $\alpha$ show very little dependence on the quartic couplings $\lambda_{i=3,4,5}$;

3. it appears that, for most parameter points, the two-loop corrections to $\alpha$ are of similar magnitude than the oneloop ones - although somewhat smaller when $\left|\lambda_{i}\right| \lesssim 1$;

4. for some parameter points, however, the two-loop corrections to $\alpha$ become significantly larger than the oneloop corrections; see the lower right plot in Fig. 7. We have verified that this happens when one of the quartic couplings $\lambda_{i}$ becomes large (typically $\left|\lambda_{i}\right| \gtrsim 1$ ) - in the plot mentioned above of $-1 / t_{\alpha}$ as a function of $\lambda_{5}$ it is $\lambda_{4}$ that becomes smaller than -1 . We may suspect the large two-loop effects are due to a loss of perturbativity: this will be discussed in more detail in the next section.

\subsection{Perturbativity constraints}

It is common in practice to use the physical scalar masses, the $\mathbb{Z}_{2}$ breaking parameter $m_{12}$ as well as the angles $\alpha, \beta$ as input for the 2HDM in numerical studies. However, this input often hides that it corresponds to huge quartic couplings which spoil unitarity and the perturbative behaviour of the theory. Therefore, the constraints that all quartics must be smaller than $4 \pi$ as well as the tree-level unitarity constraints [75-77] are applied to sort such points out. However, it was already shown in the SM that the limit of $\lambda<4 \pi$ might be too weak [78].

We now have all the machinery at hand to impose another constraint on the 2HDM model namely that the radiative corrections to the Higgs mass converge. We show here in one example that this can be a much stronger constraint than tree-level unitarity, while a more detailed analysis of this constraint on the parameter space of $2 \mathrm{HDM}$ models is left for future work.

We consider here a point for type-II defined by ${ }^{7}$

$$
\begin{aligned}
m_{H} & =593.6 \mathrm{GeV}, \quad m_{A}=535.2 \mathrm{GeV}, \\
m_{H^{+}} & =573.2 \mathrm{GeV}, \\
m_{12}^{2} & =-165675 \mathrm{GeV}^{2}, \quad \tan \alpha=-0.235, \\
\tan \beta & =1.017 .
\end{aligned}
$$

Since the masses are treated as pole masses and only treelevel relations are used in the above work, no scale for the $\overline{\mathrm{MS}}$ parameters is given. On the other side, it is usually checked that the translation of these masses into quartic couplings results in parameters which are allowed by tree-level unitarity. However, this treatment implicitly assumes that one can define at each loop level suitable counter-terms to renormalise the Higgs sector in a way that the masses can be kept constant, and that this renormalisation converges. This is, however, not the case for the parameter point defined by Eq. (6.16) as one can see as follows. The given input translates into the following set of quartic interactions using the tree-level relations ${ }^{8}$ :

$\lambda_{1}=2.831, \quad \lambda_{2}=-2.134, \quad \lambda_{3}=7.974$,

$\lambda_{4}=-0.660, \quad \lambda_{5}=0.753$

These fulfil the tree-level unitarity constraints [75-77].

To check the perturbative behaviour, we show the scale dependence in Fig. 8. Here, we used the quartic couplings of

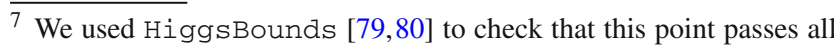
current collider limits.

${ }^{8}$ Note that negative $\lambda_{2}$ is usually taken to be forbidden because the potential is unbounded from below. However, this only holds for the tree-level potential. If RGE effects are included, $\lambda_{2}$ becomes positive after a few hundred $\mathrm{GeV}$ of running [81].
} 

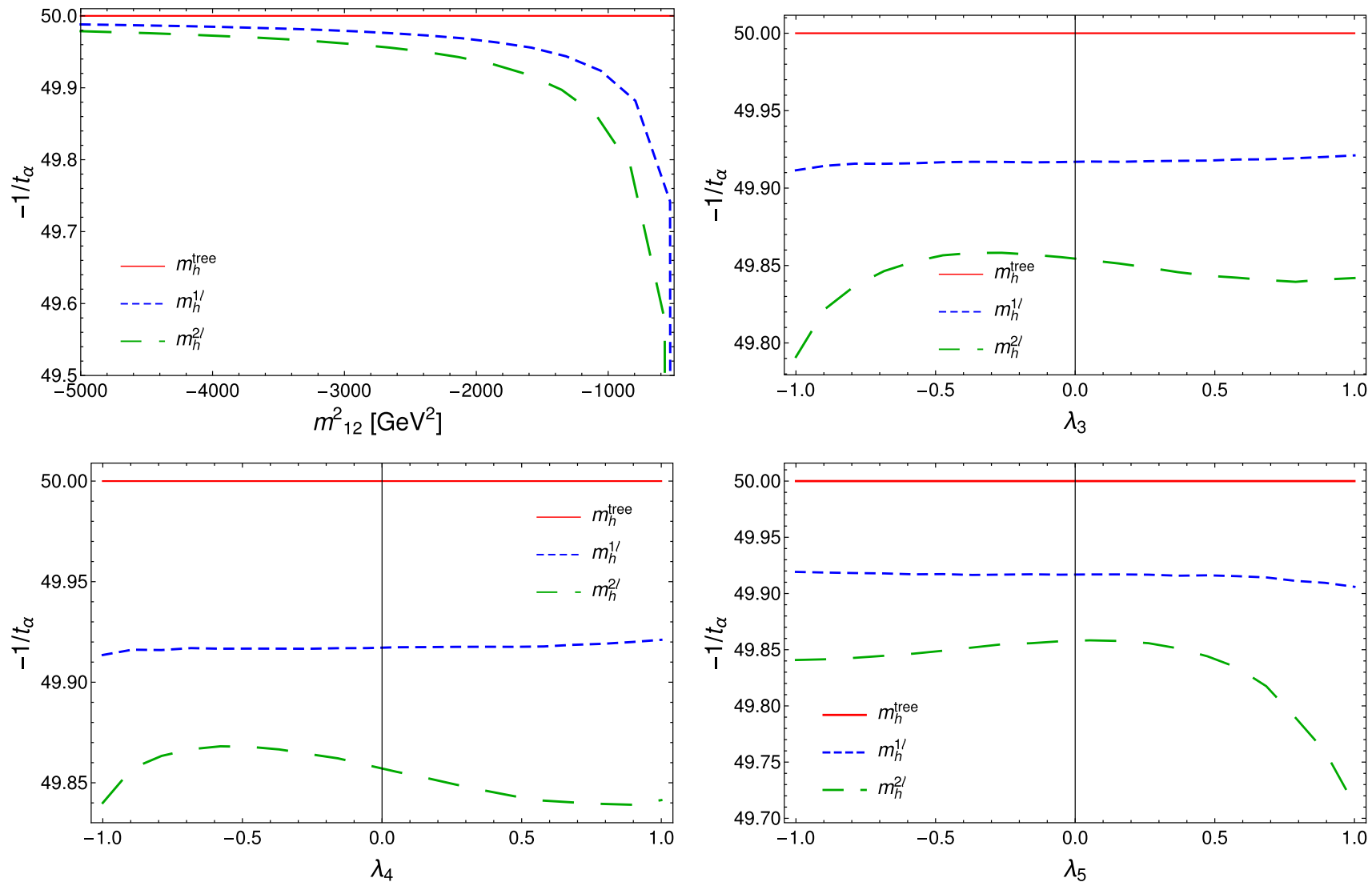

Fig. $7-1 / t_{\alpha}$ as a function of the off-diagonal mass term $m_{12}^{2}$ (upper left), and of quartic couplings $\lambda_{3}$ (upper right), $\lambda_{4}$ (lower left) and $\lambda_{5}$ (lower right) at each order in perturbation theory. For each plot we vary the parameters as follows: we choose one parameter as the abscissa; the tree-level alignment condition $\lambda_{1}=\lambda_{2}=1 / 2 \lambda_{345}$ plus the requirement that the Higgs mass is $125.09 \mathrm{GeV}$ fixes three param-

eters, namely $\lambda_{1}, \lambda_{2}$ and either $\lambda_{4}$ for the bottom right plot or $\lambda_{5}$ for the other three; the remaining parameters are held fixed at values $\lambda_{3}=0.5, \lambda_{4}=0.5, m_{12}^{2}=-1000 \mathrm{GeV}^{2}$ (when they are not otherwise varying). All plots are for $\tan \beta=50$. Red curve: tree level; blue dot-dashed curve: one-loop order; green dashed curve: two-loop order
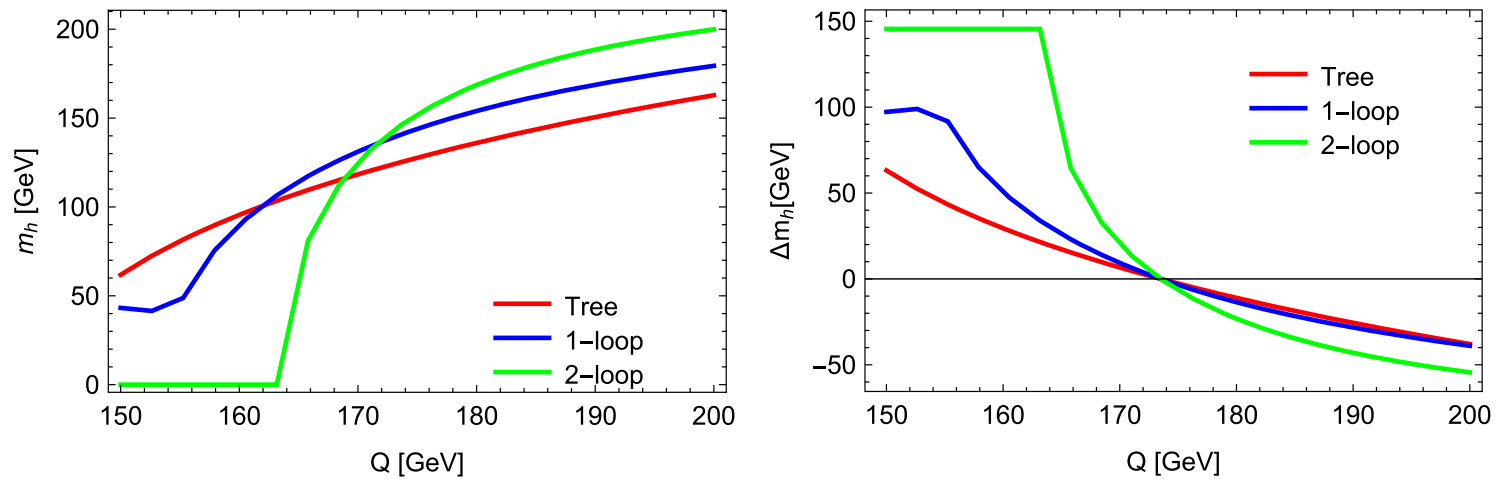

Fig. 8 The dependence of the lightest scalar mass as function of the renormalisation scale $Q$ if the quartic couplings of Eq. (6.17) are used as input at the scale $Q=m_{t}$

Eq. (6.17) as input and checked the scale dependence of the Higgs mass at different loop levels. For the evaluation of couplings to the considered scale, we used the two-loop RGEs calculated by SARAH. One sees that the scale dependence increases with increasing loop level. Of course, one might wonder if this is just an effect from our choice to define the quartic couplings at $Q=m_{t}$ as input. Therefore, we show in Fig. 9 the size of the loop corrections for different choices of our input scale $Q$. We see that the size of the loop corrections rapidly increases for $Q>m_{t}$ and the spread between one- and two-loop becomes even larger. Also choosing $Q \simeq 160 \mathrm{GeV}$ where the mass at one and two-loop level 


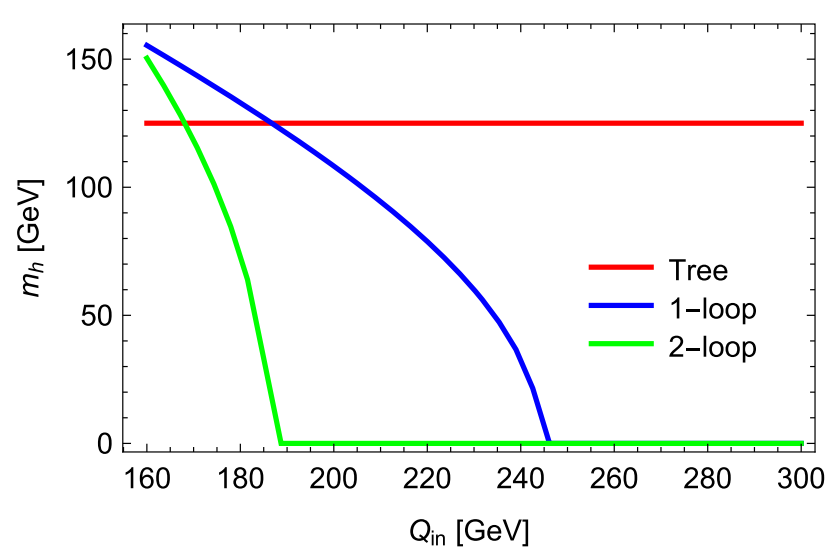

Fig. 9 The size of the one- and two-loop corrections of the lightest scalar mass as a function of the scale $Q_{i n}$ at which the input masses of Eq. (6.16) are translated into quartic couplings

seem to be roughly identical does not solve the problem: this is just a numerical coincidence and the scale dependence at two loops is even larger than at one loop.

\section{Georgi-Machacek Model}

The Georgi-Machacek Model [82] extends the SM by one real scalar $S U(2)_{L}$-triplet $\eta$ with $Y=0$ and one complex scalar $S U(2)_{L}$-triplet $\chi$ with $Y=1$, which can be written as

$$
\begin{aligned}
\eta & =\frac{1}{\sqrt{2}}\left(\begin{array}{cc}
\eta^{0} & -\sqrt{2}\left(\eta^{-}\right)^{*} \\
-\sqrt{2} \eta^{-} & -\eta^{0}
\end{array}\right), \\
\chi & =\frac{1}{\sqrt{2}}\left(\begin{array}{cc}
\chi^{-} & \sqrt{2}\left(\chi^{0}\right)^{*} \\
-\sqrt{2} \chi^{--} & -\chi^{-}
\end{array}\right) .
\end{aligned}
$$

A very compact form to write the Lagrangian in a $S U(2)_{L} \times$ $S U(2)_{R}$ invariant form is to express the doublet and triplet scalars as

$$
\Phi=\left(\begin{array}{cc}
\phi^{0 *} & \phi^{+} \\
\phi^{-} & \phi^{0}
\end{array}\right), \quad \Delta=\left(\begin{array}{ccc}
\chi^{0 *} & \eta^{+} & \chi^{++} \\
\chi^{-} & \eta^{0} & \chi^{+} \\
\chi^{--} & \eta^{-} & \chi^{0}
\end{array}\right) .
$$

Here, $\phi$ are the components of the SM doublet. Using this notation, the scalar potential reads

$$
\begin{aligned}
V(\Phi, \Delta)=\mu_{2}^{2} & \operatorname{Tr} \Phi^{\dagger} \Phi+\frac{\mu_{3}^{2}}{2} \operatorname{Tr} \Delta^{\dagger} \Delta+\lambda_{1}\left[\operatorname{Tr} \Phi^{\dagger} \Phi\right]^{2} \\
& +\lambda_{2} \operatorname{Tr} \Phi^{\dagger} \Phi \operatorname{Tr} \Delta^{\dagger} \Delta \\
& +\lambda_{3} \operatorname{Tr} \Delta^{\dagger} \Delta \Delta^{\dagger} \Delta+\lambda_{4}\left[\operatorname{Tr} \Delta^{\dagger} \Delta\right]^{2} \\
& -\lambda_{5} \operatorname{Tr}\left(\Phi^{\dagger} \sigma^{a} \Phi \sigma^{b}\right) \operatorname{Tr}\left(\Delta^{\dagger} t^{a} \Delta t^{b}\right)
\end{aligned}
$$

$$
\begin{aligned}
& -M_{1} \operatorname{Tr}\left(\Phi^{\dagger} \tau^{a} \Phi \tau^{b}\right)\left(U \Delta U^{\dagger}\right)_{a b} \\
& -M_{2} \operatorname{Tr}\left(\Delta^{\dagger} t^{a} \Delta t^{b}\right)\left(U \Delta U^{\dagger}\right)_{a b},
\end{aligned}
$$

$\tau^{a}$ and $t^{a}$ are the $S U$ (2) generators for the doublet and triplet representations, respectively, while $U$ is given for instance in [83]. The triplets obtain VEVs as

$\langle\eta\rangle=\frac{1}{\sqrt{2}}\left(\begin{array}{cc}v_{\eta} & 0 \\ 0 & -v_{\eta}\end{array}\right), \quad\langle\chi\rangle=\left(\begin{array}{cc}0 & v_{\chi} \\ 0 & 0\end{array}\right)$,

where the custodial symmetry enforces $v_{\eta}=v_{\chi} \equiv v_{T}$, and there are no tree-level contributions to the $\rho$ parameter. They further fulfil $v_{\phi}^{2}+8 v_{T}^{2}=v^{2}$, which allows us to define

$s_{H}=\sin \Theta_{H}=\frac{2 \sqrt{2} v_{T}}{v}, \quad c_{H}=\cos \Theta_{H}=\frac{v_{\phi}}{v}$.

The free parameters of the model are then

$\lambda_{1} \ldots \lambda_{5}, \quad M_{1}, \quad M_{2}, \quad s_{H}$

since $\mu_{2}^{2}, \mu_{3}^{2}$ can be eliminated by the tadpole equations. The physical eigenstates can be organised into representations of the custodial symmetry as a five-plet (consisting of a doubly charged, singly charged a neutral CP-even scalar), a triplet (consisting of a singly charged and a CP-odd neutral scalar) and two CP-even singlets (where the Standard Model Higgslike boson is the lighter of the two). Expressions for the triplet mass $m_{3}$, five-plet mass $m_{5}$ and singlet masses are given in, for example, [84].

$m_{h}, s_{H}, m_{5}$ seem to be a suitable choice for the input parameters and can be traded for $\lambda_{1}, \lambda_{5}$ and $v_{T}$. In the following we shall do this using tree-level relations derived from those in [84]. However, the choice to use masses instead of couplings as input can have the danger that one enters a nonperturbative regime without recognising it as we already have pointed out for the 2HDM. We will discuss the importance of higher-order corrections in general in this model in the following: in contrast for instance to the $2 \mathrm{HDM}$, it is not possible to renormalise all mixing angles and masses onshell in this model. One reason for this is that the masses of the five-plet are only exactly degenerate at tree level but the custodial symmetry is not protected against loop effects [85]. Therefore, the number of mass parameters but also of rotation angles is extended at the loop level: one needs three instead of two angles to diagonalise the loop-corrected CPeven mass matrix, and also the CP-odd and charged Higgs mass matrix no longer share the same angle. Therefore, an $\overline{\mathrm{MS}}$ renormalisation of the scalar sector is the natural option to check the impact of higher-order corrections to the masses 
Table 6 The scalar masses at tree and loop level for the parameter point $\lambda_{2}=\lambda_{3}=\lambda_{4}=0, m_{5}=1 \mathrm{TeV}$ and $s_{H}=0.75$. The renormalisation scale was set to $m_{5}$

\begin{tabular}{lrll}
\hline & Tree & One-loop & Two-loop \\
\hline$m_{h_{1}}(\mathrm{GeV})$ & 125.00 & 210.45 & $<0$ \\
$m_{h_{2}}(\mathrm{GeV})$ & 1000.00 & 950.56 & 916.96 \\
$m_{h_{3}}(\mathrm{GeV})$ & 1054.67 & 975.20 & 954.03 \\
$m_{A_{1}}(\mathrm{GeV})$ & 1049.31 & 998.41 & 896.13 \\
$m_{H_{1}^{+}}(\mathrm{GeV})$ & 1000.00 & 950.80 & - \\
$m_{H_{2}^{+}}(\mathrm{GeV})$ & 1049.31 & 998.21 & - \\
$m_{H^{++}}(\mathrm{GeV})$ & 1000.00 & 951.55 & - \\
\hline
\end{tabular}

and angles. We give in Table 6 the loop-corrected masses for all scalars for the parameter point $\lambda_{2}=\lambda_{3}=\lambda_{4}=0$, $m_{5}=1 \mathrm{TeV}$ and $s_{H}=0.75$.

We see in these numbers that not only a mass splitting between the components of the five- and seven-plets is induced at the one-loop level, but also that the loop corrections to the SM-like Higgs scalar can be huge. One can understand these large loop corrections for the chosen parameter point to some extent analytically: the one-loop corrections to the $(1,1)$-element of the $\mathrm{CP}$-even mass matrix are given in the effective potential in the limit $m_{5} \gg v$ by

$\Delta m_{h} \sim v^{2} \frac{8 m_{5}^{4} s_{H}^{4}}{9 \pi^{2} v^{4}}$.

Thus, for large values of $m_{5}$ and/or $s_{H}$ one can expect huge corrections to the mass. Note, there are additional loop corrections to the off-diagonal elements of the scalar mass matrix which can have a significant impact on the masses. Therefore, one needs a full numerical calculation already at the one-loop level to obtain an accurate number for the SM-like Higgs mass.

Before we further investigate the loop corrections, we want to comment briefly on the choice for the renormalisation scale $Q$. In the SM, but also in other models like 2HDMs, it is suitable to set $Q=m_{t}$ to give a good convergence and ensure that there are no large logarithmic contributions from top loops. However, in the GM model the dominant loop corrections involve often scalar fields with masses $\propto m_{5}$. Therefore, the overall size of the loop corrections is usually smaller for $Q=m_{5}$ as one can see in Fig. 10.

We check now the Higgs mass in the $\left(m_{5}, s_{H}\right)$ plane proposed in [84] always using $Q=m_{5}$. The other parameters are fixed in this plane to

$$
\begin{aligned}
m_{h}^{\text {tree }} & =125 \mathrm{GeV}, \quad M_{1}=\sqrt{2} \frac{s_{H}}{v}\left(m_{5}^{2}+v^{2}\right), \quad M_{2}=\frac{1}{6} M_{1}, \\
\lambda_{3} & =-0.1, \quad \lambda_{2}=0.4 \frac{m_{5}}{1000 \mathrm{GeV}}, \quad \lambda_{4}=0.2 .
\end{aligned}
$$

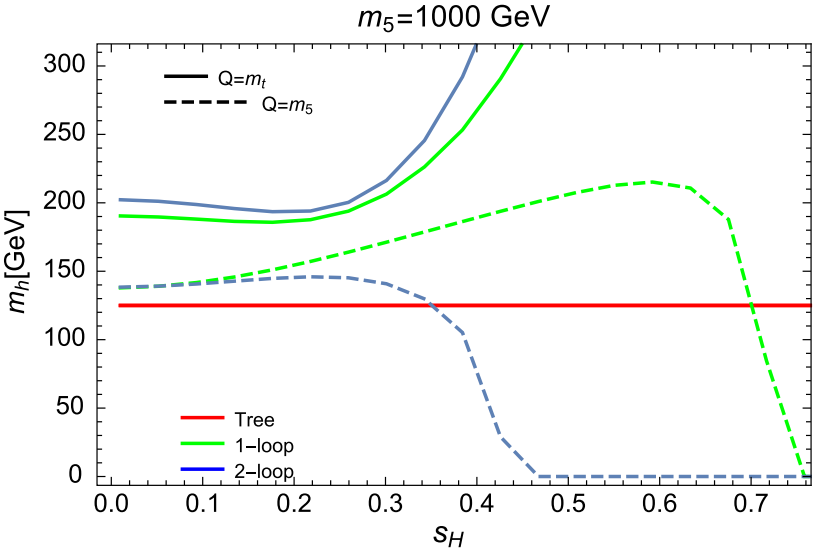

Fig. 10 The SM-like Higgs mass at tree-, one- and two-loop level for $m_{5}=1 \mathrm{TeV}$ and as a function for $s_{H}$. The results for two different choices for the renormalisation $Q$ scale are shown

The light Higgs mass at the one- and two-loop level is shown in Fig. 11. As expected, we see that the two-loop corrections are large for large $s_{H}$ and $m_{5}$. In order to further demonstrate this, we show in Fig. 11 also explicitly the size of the oneand two-loop corrections for all three CP-even scalars. We see that in the upper right corner in the $\left(s_{H}, m_{5}\right)$ plane the two-loop corrections are much larger than the one-loop ones and the Higgs can even become tachyonic. For $m_{5}=1 \mathrm{TeV}$, this already happens at $s_{H}>0.5$, while for $m_{5}=1.5 \mathrm{TeV}$ the upper limit of $s_{H}$ is as low as 0.25 . For large $m_{5}$ this limit is much stronger than the one from perturbative unitarity of $V V \rightarrow V V$ scattering amplitudes which gives $s_{H}<\frac{667 \mathrm{GeV}}{m_{5}}$ [86]. Thus, even if it might still be possible to obtain the correct Higgs mass at two-loop level by adjusting the other input parameters or by absorbing finite corrections into counter-terms, the results in this parameter region should be taken with a lot of care. Most likely, they are meaningless. However, also for the other parameter regions with a reasonable hierarchy of the one- and two-loop corrections, one would need large adjustments in the input parameters to compensate for these loop corrections. These changes would then reflect in the couplings and some decay widths of the $125-\mathrm{GeV}$ scalar will deviate for large $s_{H}$ and/or $m_{5}$ clearly from the tree-level expectation. Finally, one can also see in Fig. 12 that the loop corrections for the other scalars are sizeable and can shift the masses easily by tens to hundreds of $\mathrm{GeV}$.

\section{Conclusions}

In this paper we have presented several varied results relating to the calculation of two-loop corrections to the Higgs mass in general models. Chief among these are: 

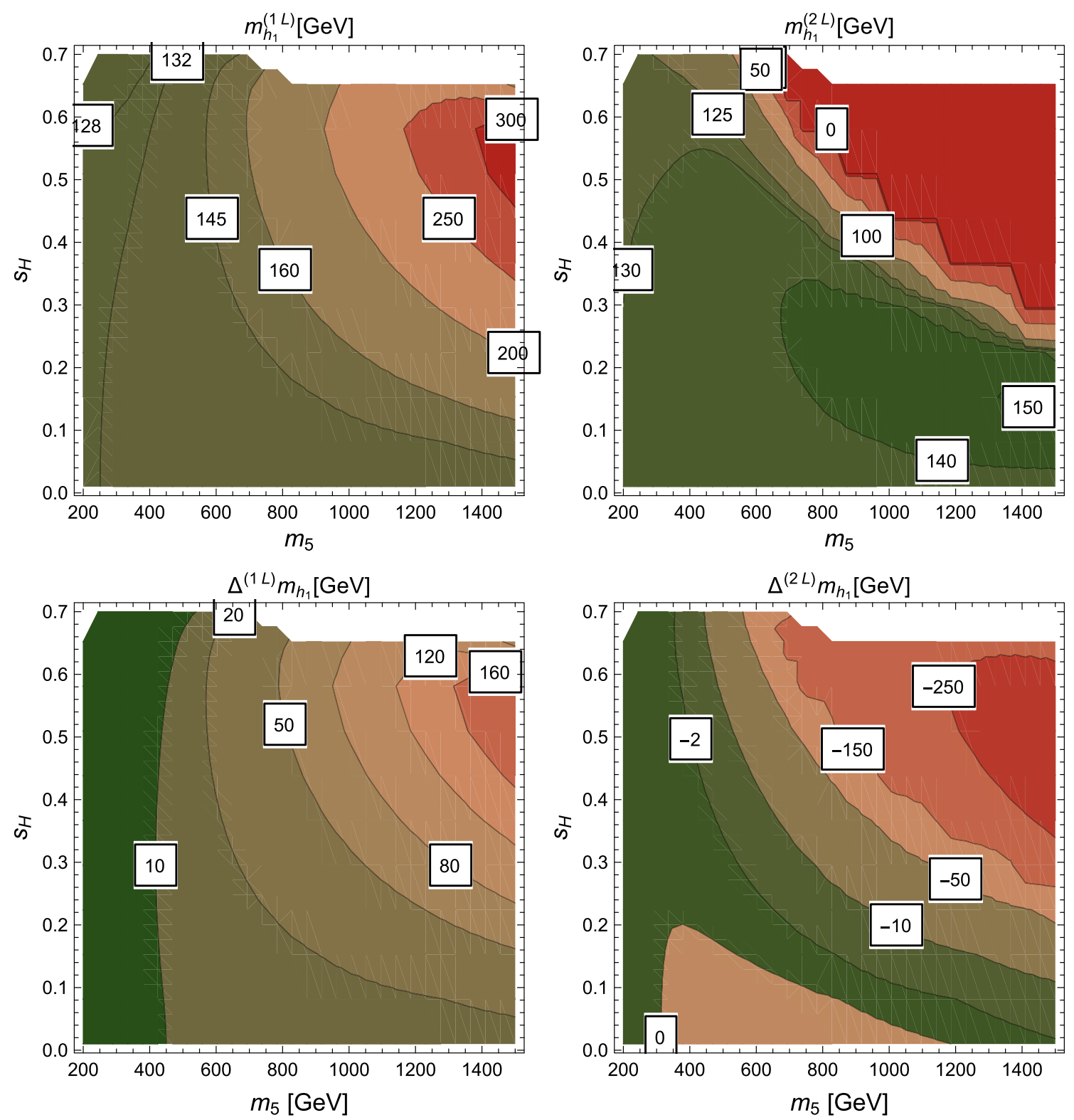

Fig. 11 First row: absolute size of the SM-like Higgs mass in the Georgi-Machacek model as a function of $s_{H}$ and $m_{5}$ at including one- (left) and two-loop (right) corrections. Second row: the size of the one- (left) and two-loop (right) corrections

1. We completed the basis of necessary loop functions for our on-shell solution, with a new expression for $\tilde{V}(0, x, y)$ given in Appendix A.

2. We extended the derivation of shifts to the tadpoles and Higgs mass from consistently solving the tadpole equations to include more general minimisation conditions, in particular allowing fermion masses to be directly dependent on the parameters (such as $\mu$ in the MSSM) with the expressions given in Appendix B.

3. We compared our results with those available for the Standard Model. In particular, this allowed a comparison within the same code of calculations in two different gauges, and we also found that the electroweak corrections are negligible, while those from momentum dependence are very small.

4. We showed that our new computation does indeed remove the instabilities (sharp peaks in the Higgs mass for certain parameter choices) in the previous approach for supersymmetric models; however, the reader should be aware that there are still some limitations when scalar masses in the loops become small compared to the renormalisation scale.

5. We explored the corrections to the mixing angle in the alignment limit in the Two Higgs Doublet Model using 

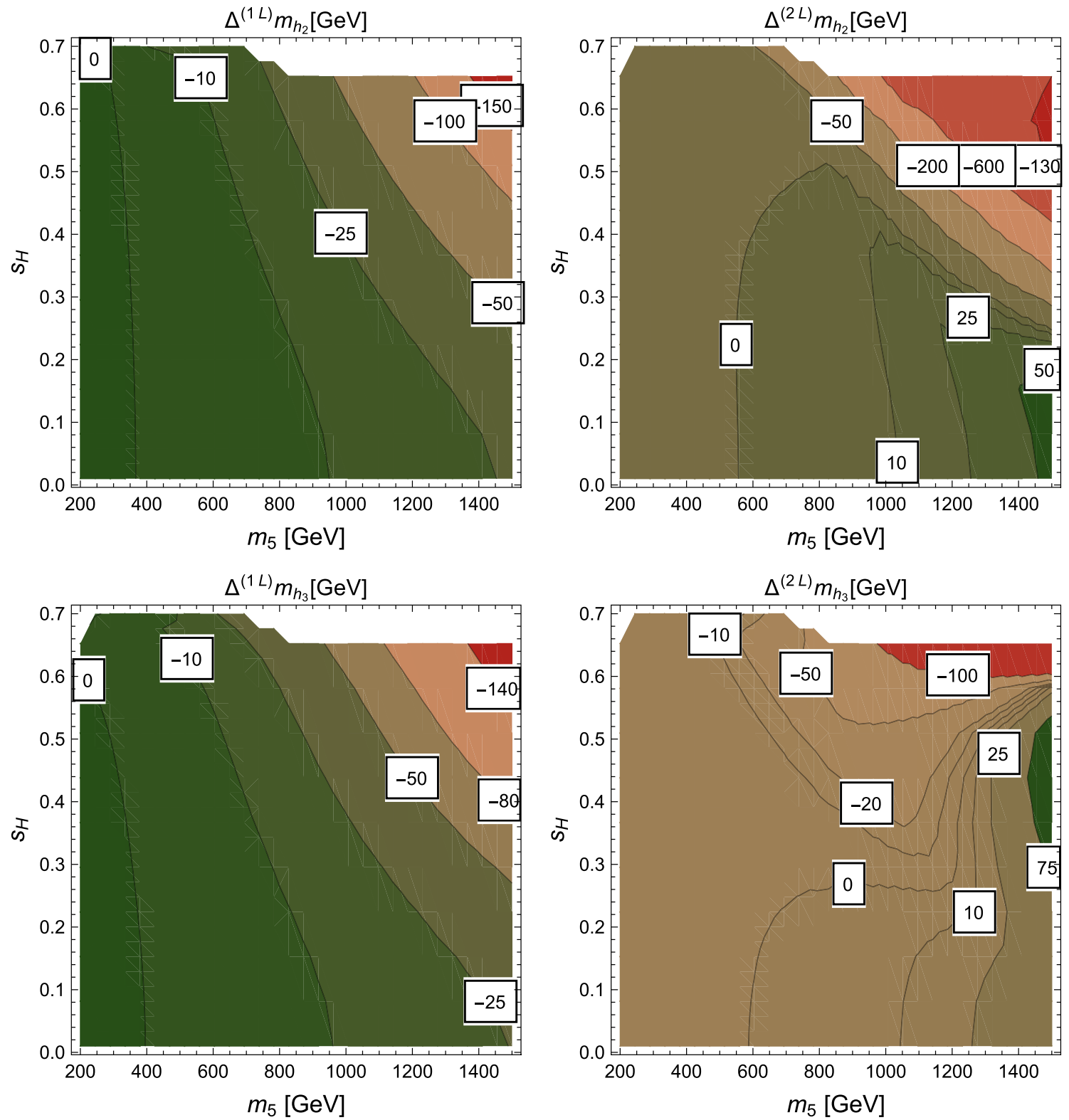

Fig. 12 The size of the one- (left) and two-loop (right) corrections in the $\left(s_{H}, m_{5}\right)$ plane for the second (first row) and third (second row) CP-even scalar

the $\overline{\mathrm{MS}}$ couplings as inputs, and found that provided the quartic couplings are chosen to be small, the loop corrections are safely under control.

6. We explored the 2HDM and Georgi-Machacek models with masses as physical inputs and using tree-level relations to obtain $\overline{\mathrm{MS}}$ couplings, as commonly done in the literature. We find that in most regions of the parameter space these lead to large quartic couplings, which rapidly lead to loss of control of the loop corrections. Perhaps surprisingly, this often occurs well before the couplings reach naive perturbativity bounds.
All of the shown results are available to the community with SARAH version 4.12.0, and we hope that this contributes to an efficient and more precise study of many extensions of the SM; this should open the avenue to much future work. It would be particularly interesting to explore more carefully the relationship between on-shell and $\overline{\mathrm{MS}}$ calculations in non-supersymmetric models, to better understand how the divergent behaviour of the masses that we observe for the $\overline{\mathrm{MS}}$ scheme translates into differences in physical couplings - or even possibly ruling out certain parameter regions of models as unphysical. 
For the technical program of generic Higgs mass computations, it would be very interesting to compute the corrections to the electroweak VEV and top Yukawa coupling to the same precision that we can achieve for the Higgs mass from $\overline{\mathrm{MS}} / \overline{\mathrm{DR}}^{\prime}$ inputs. It would also be interesting to complete the set of contributions with those stemming from electroweak couplings, even if we showed that these must be very small in the case of the Standard Model.

Acknowledgements We thank Pietro Slavich for helpful discussions and many comments on the draft. JB and MDG acknowledge support from French state funds managed by the Agence Nationale de la Recherche (ANR), in the context of the LABEX ILP (ANR-11IDEX-0004-02, ANR-10-LABX-63), and MDG acknowledges support from the ANR grant "HiggsAutomator" (ANR-15-CE31-0002). JB was supported by a scholarship from the Fondation CFM. FS is supported by ERC Recognition Award ERC-RA-0008 of the Helmholtz Association.

Open Access This article is distributed under the terms of the Creative Commons Attribution 4.0 International License (http://creativecomm ons.org/licenses/by/4.0/), which permits unrestricted use, distribution, and reproduction in any medium, provided you give appropriate credit to the original author(s) and the source, provide a link to the Creative Commons license, and indicate if changes were made. Funded by SCOAP ${ }^{3}$.

\section{A Loop functions}

A. $1 \tilde{V}(x, 0, z, u)$

One of the key functions of the basis set is $V(x, y, z, u)$. This is defined as

$V(x, y, z, u) \equiv-\frac{\partial}{\partial y} U(x, y, z, u)$.

It is singular as $y \rightarrow 0$, so we define the regularised version (defined with one fewer explicit index to [38]):

$\bar{V}(x, y, z) \equiv \lim _{u \rightarrow 0}\left[V(x, u, y, z)-\frac{1}{s-x} \frac{\partial}{\partial u} I(u, y, z)\right]$.

On the other hand, we require a slightly different regularised function:

$\tilde{V}(x, y, z) \equiv \lim _{u \rightarrow 0}\left[-V(x, u, y, z)+P_{S S}(y, z) B\left(u, x^{\prime}\right)\right]$.

For the case $x \neq 0$, we can simply extract the result at vanishing external momentum:

$$
\begin{aligned}
\lim _{s \rightarrow 0} \bar{V}(x, z, u) \\
\quad=\lim _{y \rightarrow 0}\left[-U_{0}\left(x, y^{\prime}, z, u\right)-\frac{1}{x} P_{S S}(z, u) \overline{\log } y\right] \\
=\frac{I(x, z, u)-I(0, z, u)}{x^{2}}=-\frac{1}{x} U_{0}(x, 0, z, u) .
\end{aligned}
$$

Then constructing $\tilde{V}$ gives

$$
\begin{aligned}
\lim _{s \rightarrow 0} \tilde{V}(x, z, u)= & -\lim _{s \rightarrow 0} \bar{V}(x, z, u) \\
& -\frac{1}{x}\left[R_{S S}(z, u)+P_{S S}(z, u)(\overline{\log } x-1)\right] \\
= & \frac{1}{x}\left[U_{0}(x, 0, z, u)+R_{S S}(z, u)\right. \\
& \left.+P_{S S}(z, u)(\overline{\log } x-1)\right] .
\end{aligned}
$$

On the other hand, for $x \rightarrow 0$ we cannot take the above limit. In principle we could start again from the expressions for $\bar{V}(x, y, z)$ given in the appendix of [38] and take the smooth limit $x \rightarrow 0$. Instead, here we provide two direct derivations of a compact expression for $\tilde{V}(0, z, u)$, with both general external momentum and then for our generalised effective potential limit. The starting point for the first derivation is the set of differential equations given in [39], in this case

$$
\begin{aligned}
\frac{\partial}{\partial y} & U(x, y, z, u) \\
\quad= & k_{U U} U(x, y, z, u)+k_{U T 1} T(x, z, u)+k_{U T 2} T(u, x, z) \\
& +k_{U T 2} T(z, x, u) \\
& +k_{U S}\left[S(x, z, u)-\frac{1}{2}(A(x)+A(z)+A(u)+I(y, z, u))\right] \\
& +k_{U B} B(x, y)+k_{U} \\
\equiv & k_{U U} U(x, y, z, u)+\Delta,
\end{aligned}
$$

where the coefficients of the loop functions are themselves functions of $s, x, y, z, u$. However, here we encounter the problem that several of these coefficients are actually singular as $y \rightarrow 0$ - so we cannot simply evaluate the right-hand side of the equation to determine $V(x, 0, z, u)$ !

However, we can obtain such a closed-form expression by using the ansatz

$$
\begin{aligned}
U(x, y, z, u)= & f_{0}(s ; x, z, u)+f(s ; x, z, u) A(y) \\
& +f_{1}(s ; x, z, u) y+\mathcal{O}\left(y^{2}\right) \\
f_{0}(s ; x, z, u)= & U(x, 0, z, u) \\
k_{U}= & -\frac{1}{y}+k_{U U}^{0}+\mathcal{O}(y) \\
\Delta= & \frac{\Delta^{(-1)}}{y}+\Delta_{l} \overline{\log } y+\Delta^{0}+\cdots,
\end{aligned}
$$


and substituting it into the above differential equation, to find $f$ and $f_{1}$ :

$$
\begin{aligned}
f & \overline{\log } y+f_{1}+\cdots \\
= & \left(-\frac{1}{y}+k_{U U}^{0}\right)\left(f_{0}(s ; x, z, u)+f(s ; x, z, u) A(y)\right. \\
& \left.+f_{1}(s ; x, z, u) y\right)+\Delta \\
= & -\frac{f_{0}}{y}-f \overline{\log } y+\left(f-f_{1}+f_{0} k_{U U}^{0}\right) \\
& +\frac{\Delta^{(-1)}}{y}+\Delta_{l} \overline{\log } y+\Delta^{0}+\cdots \\
& \rightarrow \Delta^{(-1)}=f_{0}, \quad f=\frac{1}{2} \Delta_{l}, \\
f_{1}= & \frac{1}{2}\left(f+\Delta^{0}+\Delta^{(-1)} k_{U U}^{0}\right) .
\end{aligned}
$$

The form of $f$ must correspond to the singularity; indeed we have

$f(s ; x, z, u)=\frac{1}{s-x} P_{S S}(z, u)$.

However, $f_{1}$ implies more work; we eventually obtain in the limit $x \rightarrow 0$, which we are interested in,

$$
\begin{aligned}
& \tilde{V}(0, z, u) \\
& =\left(\frac{u z \log z / u}{(u-z)^{3}}+\frac{u+z}{2(u-z)^{2}}\right) B(0,0) \\
& \quad+\frac{1}{s}\left[\frac{2 A(u) A(z)+(u+z)^{2}+2(u+z) I(z, u, 0)}{2(u-z)^{2}}\right] \\
& \quad+\frac{1}{2}\left[\mathcal{K}_{U T 2} T(u, 0, z)+\mathcal{K}_{U T 3} T(z, 0, u)\right. \\
& \left.\quad+\mathcal{K}_{U S} S(0, z, u)+\mathcal{K}_{U}\right]
\end{aligned}
$$

and

$$
\begin{aligned}
f_{1}(s ; 0, z, u) & =\tilde{V}(0, z, u)-\frac{P_{S S}(z, u)}{s} \overline{\log }(-s), \\
\bar{V}(0, z, u) & =-f_{1}(s ; 0, z, u)+\frac{1}{s} R_{S S}(z, u) .
\end{aligned}
$$

The coefficients defined in the above are

$$
\begin{aligned}
\mathcal{K}_{U T 2} & =-\frac{2 u z(s+u-z)}{s(u-z)^{3}} \\
\mathcal{K}_{U T 3} & =\frac{2 u z(s-u+z)}{s(u-z)^{3}} \\
\mathcal{K}_{U S} & =-\frac{2(u+z)}{s(u-z)^{2}} \\
\mathcal{K}_{U} & =-\frac{(u+z)^{2}}{(u-z)^{2}}+\frac{5(u+z)}{4(u-z)^{2}} .
\end{aligned}
$$

If we then make our generalised effective potential expansion, we find

$$
\begin{aligned}
f_{1}(s ; 0, z, u) \\
=-\frac{P_{S S}(z, u) \overline{\log }(-s)}{s} \\
\quad-\frac{\overline{\log }(-s)}{2(u-z)^{3}}\left[u^{2}-z^{2}-2 u z \log \frac{u}{z}\right] \\
\quad+\frac{1}{4(u-z)^{4}}\left[5(u+z)^{3}+8 u z I(u, z, 0)\right. \\
\quad+2 z \overline{\log } z\left(2 u^{2}-11 u z+z^{2}\right) \\
\quad+2 u \overline{\log } u\left(u^{2}-11 u z+2 z^{2}\right) \\
+4 u z(u+z) \overline{\log } u \overline{\log } z] .
\end{aligned}
$$

We do not need the limit when $u=z=0$ because in that case we have couplings $\lambda^{G G G}$. However, for $z=0$ or $u=0$ we do see that there is a smooth limit of the above.

Let us define

$f_{1}=-\frac{P_{S S}(z, u)}{s} \overline{\log }(-s)+f_{1}^{\ell} \overline{\log }(-s)+f_{1}^{0}$.

We can then write

$\tilde{V}(0, z, u)=f_{1}^{\ell} \overline{\log }(-s)+f_{1}^{0}$.

We have

$f_{1}^{\ell}(z, u)=-\frac{1}{2(u-z)^{3}}\left[u^{2}-z^{2}-2 u z \log \frac{u}{z}\right]$,

$f_{1}^{\ell}(z, z)=-\frac{1}{6 z}, \quad f_{1}^{\ell}(0, u)=-\frac{1}{2 u}$.

If we now substitute in the standard expressions for $I(z, u, 0)$ then we can simplify the above to

$$
\begin{aligned}
f_{1}^{0}(z, u)= & \frac{1}{4(u-z)^{3}} \\
\times & {\left[5\left(u^{2}-z^{2}\right)+2 z \overline{\log } z(2 u-z+u \overline{\log } z)\right.} \\
& +2 u \overline{\log } u(u-2 z-z \overline{\log } u) \\
& \left.-4 u z\left(\operatorname{Li}_{2}(1-z / u)-\operatorname{Li}_{2}(1-u / z)\right)\right]
\end{aligned}
$$

We can also write it in a shorter but less symmetric form, 


$$
\begin{aligned}
f_{1}^{0}(z, u)= & \frac{1}{4(u-z)^{3}} \\
\times & {\left[5\left(u^{2}-z^{2}\right)+2 z \overline{\log } z(2 u-z+2 u \overline{\log } u)\right.} \\
& +2 u \overline{\log } u(u-2 z-2 z \overline{\log } u) \\
& \left.-8 u z \operatorname{Li}_{2}(1-z / u)\right] .
\end{aligned}
$$

We can also take the limits:

$$
f_{1}^{0}(z, z)=\frac{11+3 \overline{\log } z}{18 z}, \quad f_{1}^{0}(0, u)=\frac{5+2 \overline{\log } u}{4 u}
$$

\section{A.1.1 Integral representation}

Our expression for $\tilde{V}$ actually lends itself for an interesting finite integral representation. We start with the definition

$\tilde{V}(0, z, u) \equiv \lim _{y \rightarrow 0}\left[-V(y, y, z, u)+B\left(s, y, y^{\prime}\right) P_{S S}(z, u)\right]$.

Then, using $C \equiv 16 \pi^{2} \frac{\mu^{2 \epsilon}}{(2 \pi)^{4-2 \epsilon}}$, we have

$$
\begin{aligned}
V & (x, y, z, u) \\
& =-\frac{\partial}{\partial y} U(x, y, z, u) \\
& =-\frac{\partial}{\partial y} \lim _{\epsilon \rightarrow 0}\left[\mathbf{U}(x, y, z, u)+1 / 2 \epsilon^{2}-1 / 2 \epsilon-\mathbf{B}(x, y) / \epsilon\right] \\
& =\lim _{\epsilon \rightarrow 0}\left[-\mathbf{U}\left(x, y^{\prime}, z, u\right)+\mathbf{B}\left(x, y^{\prime}\right) / \epsilon\right]
\end{aligned}
$$

$P_{S S}(z, u)$

$$
=-B(0 ; z, u)=-\lim _{\epsilon \rightarrow 0}[\mathbf{B}(0 ; z, u)-1 / \epsilon] \text {. }
$$

So then

$$
\begin{aligned}
V(x, y, z, u) \\
=\lim _{\epsilon \rightarrow 0}\left[C^{2} \iint \frac{1}{k^{2}+x} \frac{1}{\left((k-p)^{2}+y\right)^{2}} \frac{1}{q^{2}+z}\right. \\
\left.\quad \times \frac{1}{(q+k-p)^{2}+u}+\mathbf{B}\left(x, y^{\prime}\right) / \epsilon\right] \\
\tilde{V}(x, z, u) \\
=\lim _{y \rightarrow 0} \lim _{\epsilon \rightarrow 0}\left[-\mathbf{V}(x, y, z, u)-\mathbf{B}\left(x, y^{\prime}\right)\left(\mathbf{P}(z, u)+\frac{1}{\epsilon}\right)\right. \\
\left.\quad+\mathbf{B}\left(x, y^{\prime}\right) \mathbf{P}(z, u)+B\left(x, y^{\prime}\right) P_{S S}(z, u)\right]
\end{aligned}
$$

$$
\begin{aligned}
= & \lim _{y \rightarrow 0} \lim _{\epsilon \rightarrow 0}\left[-\mathbf{V}(x, y, z, u)+\mathbf{B}\left(x, y^{\prime}\right) \mathbf{P}(z, u)\right] \\
= & \lim _{y \rightarrow 0} \lim _{\epsilon \rightarrow 0}\left[-C^{2} \iint \frac{1}{k^{2}+x} \frac{1}{\left((k-p)^{2}+y\right)^{2}}\right. \\
& \times \frac{1}{q^{2}+z} \frac{1}{(q+k-p)^{2}+u} \\
& \left.+C^{2} \iint \frac{1}{k^{2}+x} \frac{1}{\left((k-p)^{2}+y\right)^{2}} \frac{1}{q^{2}+z} \frac{1}{q^{2}+u}\right] \\
= & \lim _{\epsilon \rightarrow 0}\left[C^{2} \iint \frac{1}{k^{2}+x} \frac{1}{\left((k-p)^{2}+y\right)^{2}} \frac{1}{q^{2}+z}\right. \\
& \left.\times \frac{2 q \cdot(k-p)+(k-p)^{2}}{\left(q^{2}+u\right)\left((q+k-p)^{2}+u\right)}\right] .
\end{aligned}
$$

We can then integrate this expression. For the case $x \rightarrow 0$ we can simplify a little:

$$
\begin{aligned}
\tilde{V}(0, z, u)= & \lim _{y \rightarrow 0} \lim _{\epsilon \rightarrow 0}\left[C^{2} \iint \frac{1}{(k+p)^{2}} \frac{1}{\left(k^{2}+y\right)^{2}} \frac{1}{q^{2}+z}\right. \\
& \left.\times \frac{2 q \cdot k+k^{2}}{\left(q^{2}+u\right)\left((q+k)^{2}+u\right)}\right] \\
\equiv & \lim _{\epsilon \rightarrow 0}\left(-\frac{1}{z-u} \mathbf{F}(z, u)\right) \\
\mathbf{F}(z, u) \equiv & C^{2} \iint \frac{1}{(k+p)^{2}} \frac{1}{k^{4}} \frac{1}{q^{2}+z} \frac{2 q \cdot k+k^{2}}{(q+k)^{2}+u} .
\end{aligned}
$$

This integral is finite; we have checked that explicitly performing the integral using TARCER [60] exactly yields Eq. (A.10).

\section{A.2 Limits of $M(0, y, 0, u, v)$}

Here we shall give explicit limits of the M function:

$$
M(0, y, 0, u, v)=A_{M}(y, u, v) \overline{\log }(-s)+B_{M}(y, u, v),
$$

$$
\begin{aligned}
& A_{M}(y, u, v)=\frac{u \overline{\log } u}{(y-u)(u-v)}-\frac{y \overline{\log } y}{(y-u)(y-v)} \\
& -\frac{v \overline{\log } v}{(y-v)(u-v)}, \\
& B_{M}(y, u, v)=-(2+\overline{\log } v) A_{M}(y, u, v) \\
& +\frac{u+v}{(y-u)(u-v)} \operatorname{Li}_{2}(1-u / v) \\
& -\frac{v+y}{(y-u)(y-v)} \mathrm{Li}_{2}(1-y / v) \text {. }
\end{aligned}
$$

$A_{M}$ is symmetric on all three indices, and as we already have an expression for $M(0,0,0,0, v)$ [37], and as $M(0,0,0, u, 0)$ or $M(0, y, 0,0,0)$ has a pre-factor $\lambda^{G G G}$, we only need to consider the following cases: 


$$
\begin{aligned}
& A_{M}(0, u, v)=\frac{\overline{\log }(v / u)}{u-v}, \\
& B_{M}(0, u, v)=-(2+\overline{\log } v) A_{M}(0, u, v)-\frac{\pi^{2}}{6 u} \\
& -\frac{(u+v) \operatorname{Li}_{2}(1-u / v)}{u(u-v)}, \\
& B_{M}(y, u, 0)=\frac{\log u / y[4+\overline{\log } u+\overline{\log } y]}{2(u-y)}, \\
& B_{M}(y, y, 0)=\frac{2+\overline{\log } y}{y}, \\
& A_{M}(y, y, v)=\frac{v \log y / v}{(y-v)^{2}}-\frac{1}{y-v}, \\
& A_{M}(y, y, 0)=-\frac{1}{y}, \\
& B_{M}(y, y, v)=-(2+\overline{\log } v) A_{M}(y, y, v)+\frac{1}{(y-v)^{2}} \\
& \times\left[(v+y) \log y / v+2 v \operatorname{Li}_{2}(1-y / v)\right], \\
& A_{M}(y, y, y)=-\frac{1}{2 y} \text {, } \\
& B_{M}(y, y, y)=\frac{1}{2 y}(3+\overline{\log }(y)) \\
& =-(2+\overline{\log } y) A_{M}(y, y, y)+\frac{1}{2 y}, \\
& B_{M}(y, u, u)=-(2+\overline{\log } u) A_{M}(y, u, u)+\frac{2}{u-y} \\
& -\frac{(u+y) \operatorname{Li}_{2}(1-y / u)}{(u-y)^{2}} \text {. }
\end{aligned}
$$

\section{B Consistent solution of the tadpole equations with shifts to fermion masses}

Here we give the two-loop shifts to the tadpoles and selfenergies due to shifts in fermion masses when we solve the tadpole equations consistently.

We denote the undiagonalised fermion mass matrix as $m^{I J}$. The mass-squared matrix is defined [58] as

$$
\left(m^{2}\right)_{I}^{J}=m_{I K}^{*} m^{K J}
$$

and it is diagonalised by a unitary matrix $N$ defined such that

$$
\begin{aligned}
m_{I}^{2} \delta_{I}^{J} & =N_{I}^{K} N_{L}^{* J}\left(m^{2}\right)_{K}^{L}, \\
M^{I J} & \equiv N_{K}^{*}{ }^{I} N_{L}^{*}{ }^{J} m^{K L} \\
\rightarrow M^{I K} M_{J K} & =m_{I}^{2} \delta_{I}{ }^{J} .
\end{aligned}
$$

Then if the tree-level matrices depend on some parameters $\left\{x_{i}\right\}$ for which we solve the tadpole equations as in equation
(2.7) we have

$\delta M^{I J}=N_{K}^{* I} \frac{\partial m^{K L}}{\partial x_{k}} N_{L}^{*} J c_{k l} \frac{\partial \Delta V^{(1)}}{\partial \phi_{l}^{0}}$.

Then the shift to the fermion contribution to the tadpole is

$$
\begin{aligned}
\delta^{(2)} & \left(\left.\frac{\partial V_{F}^{(1)}}{\partial \phi_{r}^{0}}\right|_{\varphi=v}\right) \\
= & -R_{r p} \operatorname{Re}\left[y^{K L p} \delta M_{K L}^{*}\right]\left(A\left(m_{K}^{2}\right)+A\left(m_{L}^{2}\right)\right) \\
& -2 R_{r p} \operatorname{Re}\left[Y^{I J p} M_{J K}^{*}\right]\left(\delta M^{K L} M_{I L}^{*}+\delta M_{I L}^{*} M^{K L}\right) \\
& \times P_{S S}\left(m_{I}^{2}, m_{K}^{2}\right),
\end{aligned}
$$

while the shift to the scalar self-energy is

$$
\begin{aligned}
\delta \Pi_{i j}^{(2), F}= & -2 \operatorname{Re}\left[y^{K L i} y_{K^{\prime} L j}\right] \\
& \times\left(M_{K J}^{*} \delta M^{K^{\prime} J}+\delta M_{K J^{*}}^{*} \bar{M}^{K^{\prime} J}\right) \\
& \times\left[P_{S S}\left(m_{K}^{2}, m_{K^{\prime}}^{2}\right)-B\left(m_{K^{\prime}}^{2}, m_{L}^{2}\right)\right. \\
& -\left(m_{K}^{2}+m_{K^{\prime}}^{2}-s\right) \\
& \left.\times C\left(s, s, 0, m_{K}^{2}, m_{L}^{2}, m_{K^{\prime}}^{2}\right)\right] \\
& +4 \operatorname{Re}\left[y^{K L i} y^{K^{\prime} L^{\prime} j} \delta M_{K K^{\prime}}^{*} M_{L L^{\prime}}^{*}\right] B\left(m_{K}^{2}, m_{L}^{2}\right) \\
& +4 \operatorname{Re}\left[y^{K L i} y^{K^{\prime} L^{\prime} j} M_{I K^{\prime}}^{*} M_{L L^{\prime}}^{*}\right] \\
& \times\left(M_{K J}^{*} \delta M^{I J}+\delta M_{K J}^{*} M^{I J}\right) \\
& \times C\left(s, s, 0, m_{K}^{2}, m_{L}^{2}, m_{I}^{2}\right) .
\end{aligned}
$$

To illustrate this, consider the MSSM, where the tadpole equations read

$$
\begin{aligned}
& \left(|\mu|^{2}+m_{H_{u}}^{2}\right) v_{u}-B_{\mu} v_{d}+\frac{1}{8}\left(g_{Y}^{2}+g_{2}^{2}\right)\left(v_{u}^{2}-v_{d}^{2}\right) v_{u} \\
& =-\frac{\partial \Delta V}{\partial v_{u}} \\
& \left(|\mu|^{2}+m_{H_{d}}^{2}\right) v_{d}-B_{\mu} v_{u}-\frac{1}{8}\left(g_{Y}^{2}+g_{2}^{2}\right)\left(v_{u}^{2}-v_{d}^{2}\right) v_{d} \\
& =-\frac{\partial \Delta V}{\partial v_{d}} .
\end{aligned}
$$

Solving for $|\mu|^{2}$ we have

$$
\begin{aligned}
|\mu|^{2}= & -\frac{M_{Z}^{2}}{2}+\frac{1}{c_{2 \beta}} \\
& \times\left[m_{H_{u}}^{2} s_{\beta}^{2}-m_{H_{d}}^{2} c_{\beta}^{2}+\frac{1}{v} s_{\beta} \frac{\partial \Delta V}{\partial v_{u}}-\frac{1}{v} c_{\beta} \frac{\partial \Delta V}{\partial v_{d}}\right],
\end{aligned}
$$


so we have

$\delta \mu=\frac{1}{2 \mu^{*} v c_{2 \beta}}\left[s_{\beta} \frac{\partial \Delta V}{\partial v_{u}}-c_{\beta} \frac{\partial \Delta V}{\partial v_{d}}\right]$.

This in turn will lead to a shift in the neutralino and chargino masses, which lead to a shift to the two-loop tadpoles.

\section{References}

1. H.E. Haber, R. Hempfling, Can the mass of the lightest Higgs boson of the minimal supersymmetric model be larger than $\mathrm{m}(\mathrm{Z})$ ? Phys. Rev. Lett. 66, 1815-1818 (1991)

2. Y. Okada, M. Yamaguchi, T. Yanagida, Upper bound of the lightest Higgs boson mass in the minimal supersymmetric standard model. Prog. Theor. Phys. 85, 1-6 (1991)

3. J.R. Ellis, G. Ridolfi, F. Zwirner, On radiative corrections to supersymmetric Higgs boson masses and their implications for LEP searches. Phys. Lett. B 262, 477-484 (1991)

4. R. Hempfling, A.H. Hoang, Two loop radiative corrections to the upper limit of the lightest Higgs boson mass in the minimal supersymmetric model. Phys. Lett. B 331, 99-106 (1994). arXiv:hep-ph/9401219

5. S. Heinemeyer, W. Hollik, G. Weiglein, QCD corrections to the masses of the neutral CP-even Higgs bosons in the MSSM. Phys. Rev. D 58, 091701 (1998). arXiv:hep-ph/9803277

6. S. Heinemeyer, W. Hollik, G. Weiglein, Precise prediction for the mass of the lightest Higgs boson in the MSSM. Phys. Lett. B 440, 296-304 (1998). arXiv:hep-ph/9807423

7. R.-J. Zhang, Two loop effective potential calculation of the lightest CP even Higgs boson mass in the MSSM. Phys. Lett. B 447, 89-97 (1999). arXiv:hep-ph/9808299

8. S. Heinemeyer, W. Hollik, G. Weiglein, The Masses of the neutral CP - even Higgs bosons in the MSSM: Accurate analysis at the two loop level. Eur. Phys. J. C 9, 343-366 (1999). arXiv:hep-ph/9812472

9. J.R. Espinosa, R.-J. Zhang, MSSM lightest CP even Higgs boson mass to $\mathrm{O}(\mathrm{alpha}(\mathrm{s})$ alpha(t)): The effective potential approach. JHEP 03, 026 (2000). arXiv:hep-ph/9912236

10. J.R. Espinosa, R.-J. Zhang, Complete two loop dominant corrections to the mass of the lightest CP even Higgs boson in the minimal supersymmetric standard model. Nucl. Phys. B 586, 3-38 (2000). arXiv:hep-ph/0003246

11. G. Degrassi, P. Slavich, F. Zwirner, On the neutral Higgs boson masses in the MSSM for arbitrary stop mixing. Nucl. Phys. B 611, 403-422 (2001). arXiv:hep-ph/0105096

12. A. Brignole, G. Degrassi, P. Slavich, F. Zwirner, On the $\mathrm{O}($ alpha(t)**2) two loop corrections to the neutral Higgs boson masses in the MSSM. Nucl. Phys. B 631, 195-218 (2002). arXiv:hep-ph/0112177

13. A. Brignole, G. Degrassi, P. Slavich, F. Zwirner, On the two loop sbottom corrections to the neutral Higgs boson masses in the MSSM. Nucl. Phys. B 643, 79-92 (2002). arXiv:hep-ph/0206101

14. S.P. Martin, Two loop effective potential for the minimal supersymmetric standard model. Phys. Rev. D 66, 096001 (2002). arXiv:hep-ph/0206136

15. S.P. Martin, Complete two loop effective potential approximation to the lightest Higgs scalar boson mass in supersymmetry. Phys. Rev. D 67, 095012 (2003). arXiv:hep-ph/0211366

16. A. Dedes, G. Degrassi, P. Slavich, On the two loop Yukawa corrections to the MSSM Higgs boson masses at large tan beta. Nucl. Phys. B 672, 144-162 (2003). arXiv:hep-ph/0305127
17. S. Heinemeyer, W. Hollik, H. Rzehak, G. Weiglein, High-precision predictions for the MSSM Higgs sector at O(alpha(b) alpha(s)). Eur. Phys. J. C 39, 465-481 (2005). arXiv:hep-ph/0411114

18. S.P. Martin, Three-loop corrections to the lightest Higgs scalar boson mass in supersymmetry. Phys. Rev. D 75, 055005 (2007). arXiv:hep-ph/0701051

19. R.V. Harlander, P. Kant, L. Mihaila, M. Steinhauser, Higgs boson mass in supersymmetry to three loops. Phys. Rev. Lett. 100, 191602 (2008). arXiv:0803.0672 [hep-ph]

20. R.V. Harlander, P. Kant, L. Mihaila, M. Steinhauser, Higgs boson mass in supersymmetry to three loops. Phys. Rev. Lett. 101, 039901 (2008)

21. P. Kant, R.V. Harlander, L. Mihaila, M. Steinhauser, Light MSSM Higgs boson mass to three-loop accuracy. JHEP 08, 104 (2010). arXiv: 1005.5709 [hep-ph]

22. W. Hollik, S. Paßehr, Two-loop top-Yukawa-coupling corrections to the Higgs boson masses in the complex MSSM. Phys. Lett. B 733, 144-150 (2014). arXiv:1401.8275 [hep-ph]

23. W. Hollik, S. Paßehr, Higgs boson masses and mixings in the complex MSSM with two-loop top-Yukawa-coupling corrections. JHEP 10, 171 (2014). arXiv:1409.1687 [hep-ph]

24. S. Paßehr, G. Weiglein, Two-loop top and bottom Yukawa corrections to the Higgs-boson masses in the complex MSSM. arXiv:1705.07909 [hep-ph]

25. S.P. Martin, Strong and Yukawa two-loop contributions to Higgs scalar boson self-energies and pole masses in supersymmetry. Phys. Rev. D 71, 016012 (2005). arXiv:hep-ph/0405022

26. S. Borowka, T. Hahn, S. Heinemeyer, G. Heinrich, W. Hollik, Momentum-dependent two-loop QCD corrections to the neutral Higgs-boson masses in the MSSM. Eur. Phys. J. C 74(8), 2994 (2014). arXiv:1404.7074 [hep-ph]

27. G. Degrassi, S. Di Vita, P. Slavich, Two-loop QCD corrections to the MSSM Higgs masses beyond the effective-potential approximation. Eur. Phys. J. C 75(2), 61 (2015). arXiv:1410.3432 [hep-ph]

28. S.P. Martin, Taming the Goldstone contributions to the effective potential. Phys. Rev. D 90(1), 016013 (2014). arXiv:1406.2355 [hep-ph]

29. J. Elias-Miro, J.R. Espinosa, T. Konstandin, Taming infrared divergences in the effective potential. JHEP 08, 034 (2014). arXiv: 1406.2652 [hep-ph]

30. A. Andreassen, W. Frost, M.D. Schwartz, Consistent use of effective potentials. Phys. Rev. D 91(1), 016009 (2015). arXiv:1408.0287 [hep-ph]

31. J.R. Espinosa, M. Garny, T. Konstandin, A. Riotto, Gaugeindependent scales related to the standard model vacuum instability. arXiv:1608.06765 [hep-ph]

32. J.R. Espinosa, M. Garny, T. Konstandin, Interplay of infrared divergences and gauge-dependence of the effective potential. arXiv:1607.08432 [hep-ph]

33. N. Kumar, S.P. Martin, Resummation of Goldstone boson contributions to the MSSM effective potential. Phys. Rev. D 94(1), 014013 (2016). arXiv:1605.02059 [hep-ph]

34. A. Pilaftsis, D. Teresi, Symmetry improved CJT effective action. Nucl. Phys. B 874(2), 594-619 (2013). arXiv:1305.3221 [hep-ph]

35. A. Pilaftsis, D. Teresi, Symmetry improved 2PI effective action and the infrared divergences of the standard model. J. Phys. Conf. Ser. 631(1), 012008 (2015). arXiv:1502.07986 [hep-ph]

36. A. Pilaftsis, D. Teresi, Symmetry-improved 2PI approach to the Goldstone-Boson IR problem of the SM effective potential. Nucl. Phys. B 906, 381-407 (2016). arXiv:1511.05347 [hep-ph]

37. J. Braathen, M.D. Goodsell, Avoiding the Goldstone Boson catastrophe in general renormalisable field theories at two loops. JHEP 12, 056 (2016). arXiv:1609.06977 [hep-ph]

38. S.P. Martin, Two loop scalar self energies in a general renormalizable theory at leading order in gauge couplings. Phys. Rev. D 70, 016005 (2004). arXiv:hep-ph/0312092 
39. S.P. Martin, Evaluation of two loop selfenergy basis integrals using differential equations. Phys. Rev. D 68, 075002 (2003). arXiv:hep-ph/0307101

40. S.P. Martin, Two-loop scalar self-energies and pole masses in a general renormalizable theory with massless gauge bosons. Phys. Rev. D 71, 116004 (2005). arXiv:hep-ph/0502168

41. M. Goodsell, K. Nickel, F. Staub, Generic two-loop Higgs mass calculation from a diagrammatic approach. Eur. Phys. J. C 75(6), 290 (2015). arXiv:1503.03098 [hep-ph]

42. F. Staub, SARAH. arXiv:0806.0538 [hep-ph]

43. F. Staub, From Superpotential to Model Files for FeynArts and CalcHep/CompHep. Comput. Phys. Commun. 181, 1077-1086 (2010). arXiv:0909.2863 [hep-ph]

44. F. Staub, Automatic calculation of supersymmetric renormalization group equations and self energies. Comput. Phys. Commun. 182, 808-833 (2011). arXiv:1002.0840 [hep-ph]

45. F. Staub, SARAH 3.2: Dirac Gauginos, UFO output, and more. Comput. Phys. Commun. 184, 1792-1809 (2013). arXiv:1207.0906 [hep-ph]

46. F. Staub, SARAH 4: A tool for (not only SUSY) model builders. Comput. Phys. Commun. 185, 1773-1790 (2014). arXiv:1309.7223 [hep-ph]

47. F. Staub, Exploring new models in all detail with SARAH. Adv. High Energy Phys. 2015, 840780 (2015). arXiv:1503.04200 [hep$\mathrm{ph}]$

48. W. Porod, SPheno, a program for calculating supersymmetric spectra, SUSY particle decays and SUSY particle production at e+ e- colliders. Comput. Phys. Commun. 153, 275-315 (2003). arXiv:hep-ph/0301101

49. W. Porod, F. Staub, SPheno 3.1: Extensions including flavour, CPphases and models beyond the MSSM. Comput. Phys. Commun. 183, 2458-2469 (2012). arXiv:1104.1573 [hep-ph]

50. M.D. Goodsell, K. Nickel, F. Staub, Two-Loop Higgs mass calculations in supersymmetric models beyond the MSSM with SARAH and SPheno. Eur. Phys. J. C 75(1), 32 (2015). arXiv:1411.0675 [hep-ph]

51. M.D. Goodsell, F. Staub, The Higgs mass in the CP violating MSSM, NMSSM, and beyond. arXiv:1604.05335 [hep-ph]

52. M.D. Goodsell, M.E. Krauss, T. Müller, W. Porod, F. Staub, Dark matter scenarios in a constrained model with Dirac gauginos. JHEP 10, 132 (2015). arXiv:1507.01010 [hep-ph]

53. K. Benakli, L. Darmé, M.D. Goodsell, J. Harz, The Di-Photon Excess in a Perturbative SUSY Model. Nucl. Phys. B 911, 127162 (2016). arXiv:1605.05313 [hep-ph]

54. P. Athron, J.-H. Park, T. Steudtner, D. Stöckinger, A. Voigt, Precise Higgs mass calculations in (non-)minimal supersymmetry at both high and low scales. arXiv:1609.00371 [hep-ph]

55. S.P. Martin, D.G. Robertson, Higgs boson mass in the Standard Model at two-loop order and beyond. Phys. Rev. D 90(7), 073010 (2014). arXiv:1407.4336 [hep-ph]

56. D. Buttazzo, G. Degrassi, P.P. Giardino, G.F. Giudice, F. Sala, A. Salvio, A. Strumia, Investigating the near-criticality of the Higgs boson. JHEP 12, 089 (2013). arXiv:1307.3536 [hep-ph]

57. J. Braathen, M.D. Goodsell, P. Slavich, Leading two-loop corrections to the Higgs boson masses in SUSY models with Dirac gauginos. JHEP 09, 045 (2016). arXiv: 1606.09213 [hep-ph]

58. S.P. Martin, Two loop effective potential for a general renormalizable theory and softly broken supersymmetry. Phys. Rev. D 65 , 116003 (2002). arXiv:hep-ph/0111209

59. S.P. Martin, D.G. Robertson, TSIL: A Program for the calculation of two-loop self-energy integrals. Comput. Phys. Commun. 174, 133-151 (2006). arXiv:hep-ph/0501132

60. R. Mertig, R. Scharf, TARCER: A Mathematica program for the reduction of two loop propagator integrals. Comput. Phys. Commun. 111, 265-273 (1998). arXiv:hep-ph/9801383
61. F. Staub, W. Porod, Improved predictions for intermediate and heavy supersymmetry in the MSSM and beyond. Eur. Phys. J. C 77(5), 338 (2017). arXiv:1703.03267 [hep-ph]

62. U. Ellwanger, C. Hugonie, A.M. Teixeira, The next-to-minimal supersymmetric standard model. Phys. Rept. 496, 1-77 (2010). arXiv:0910.1785 [hep-ph]

63. N. Arkani-Hamed, S. Dimopoulos, Supersymmetric unification without low energy supersymmetry and signatures for fine-tuning at the LHC. JHEP 06, 073 (2005). arXiv:hep-th/0405159

64. G.F. Giudice, A. Romanino, Split supersymmetry. Nucl. Phys. B 699, 65-89 (2004). arXiv:hep-ph/0406088 [Erratum: Nucl. Phys. B 706, 487 (2005)]

65. N. Arkani-Hamed, S. Dimopoulos, G.F. Giudice, A. Romanino, Aspects of split supersymmetry. Nucl. Phys. B709, 3-46 (2005). arXiv:hep-ph/0409232

66. W. Kilian, T. Plehn, P. Richardson, E. Schmidt, Split supersymmetry at colliders. Eur. Phys. J. C 39, 229-243 (2005). arXiv:hep-ph/0408088

67. N. Bernal, A. Djouadi, P. Slavich, The MSSM with heavy scalars. JHEP 07, 016 (2007). arXiv:0705.1496 [hep-ph]

68. G.F. Giudice, A. Strumia, Probing high-scale and split supersymmetry with higgs mass measurements. Nucl. Phys. B 858, 63-83 (2012). arXiv:1108.6077 [hep-ph]

69. E. Bagnaschi, G.F. Giudice, P. Slavich, A. Strumia, Higgs mass and unnatural supersymmetry. JHEP 09, 092 (2014). arXiv:1407.4081 [hep-ph]

70. J. Pardo Vega, G. Villadoro, SusyHD: Higgs mass determination in supersymmetry. JHEP 07, 159 (2015). arXiv:1504.05200 [hep-ph]

71. E. Bagnaschi, J. Pardo Vega, P. Slavich, Improved determination of the Higgs mass in the MSSM with heavy superpartners. Eur. Phys. J. C 77(5), 334 (2017). arXiv:1703.08166 [hep-ph]

72. K. Benakli, L. Darmé, M.D. Goodsell, P. Slavich, A fake split supersymmetry model for the $126 \mathrm{GeV}$ Higgs. JHEP 05, 113 (2014). arXiv:1312.5220 [hep-ph]

73. K. Benakli, L. Darmé, M.D. Goodsell, (O)Mega split. JHEP 11, 100 (2015). arXiv:1508.02534 [hep-ph]

74. J. Bernon, J.F. Gunion, H.E. Haber, Y. Jiang, S. Kraml, Scrutinizing the alignment limit in two-Higgs-doublet models: $\mathrm{mh}=125 \mathrm{GeV}$. Phys. Rev. D 92(7), 075004 (2015). arXiv:1507.00933 [hep-ph]

75. S. Kanemura, T. Kubota, E. Takasugi, Lee-Quigg-Thacker bounds for Higgs boson masses in a two doublet model. Phys. Lett. B 313, 155-160 (1993). arXiv:hep-ph/9303263

76. A.G. Akeroyd, A. Arhrib, E.-M. Naimi, Note on tree level unitarity in the general two Higgs doublet model. Phys. Lett. B 490, 119-124 (2000). arXiv:hep-ph/0006035

77. J. Horejsi, M. Kladiva, Tree-unitarity bounds for THDM Higgs masses revisited. Eur. Phys. J. C 46, 81-91 (2006). arXiv:hep-ph/0510154

78. U. Nierste, K. Riesselmann, Higgs sector renormalization group in the MS and OMS scheme: The Breakdown of perturbation theory for a heavy Higgs. Phys. Rev. D 53, 6638-6652 (1996). arXiv:hep-ph/9511407

79. P. Bechtle, O. Brein, S. Heinemeyer, G. Weiglein, K.E. Williams, HiggsBounds: Confronting arbitrary Higgs sectors with exclusion bounds from LEP and the tevatron. Comput. Phys. Commun. 181, 138-167 (2010). arXiv:0811.4169 [hep-ph]

80. P. Bechtle, O. Brein, S. Heinemeyer, O. Stål, T. Stefaniak, G. Weiglein, K.E. Williams, HiggsBounds - 4: Improved tests of extended Higgs sectors against exclusion bounds from LEP, the tevatron and the LHC. Eur. Phys. J. C 74(3), 2693 (2014). arXiv:1311.0055 [hep-ph]

81. F. Staub, Reopen parameter regions in Two-Higgs Doublet Models. arXiv:1705.03677 [hep-ph]

82. H. Georgi, M. Machacek, Doubly charged Higgs Bosons. Nucl. Phys. B262, 463-477 (1985) 
83. K. Hartling, K. Kumar, H.E. Logan, The decoupling limit in the Georgi-Machacek model. Phys. Rev. D 90(1), 015007 (2014). arXiv:1404.2640 [hep-ph]

84. LHC Higgs Cross Section Working Group Collaboration, D. de Florian et al., Handbook of LHC Higgs Cross Sections: 4. Deciphering the nature of the Higgs sector. arXiv:1610.07922 [hep-ph]
85. S. Blasi, S. De Curtis, K. Yagyu, Effects of custodial symmetry breaking in the Georgi-Machacek model at high energies. arXiv: 1704.08512 [hep-ph]

86. H.E. Logan, V. Rentala, All the generalized Georgi-Machacek models. Phys. Rev. D 92(7), 075011 (2015). arXiv:1502.01275 [hep-ph] 